JOURNAL OF INTEGRAL EQUATIONS AND APPLICATIONS

Volume 7, Number 2, Spring 1995

\title{
INTEGRATED RESOLVENT OPERATORS
}

\author{
HIROKAZU OKA
}

ABSTRACT. In this paper we introduce the notion of integrated resolvent operators to study the linear Volterra integrodifferential equation

$$
\begin{gathered}
u^{\prime}(t)=A u(t)+\int_{0}^{t} B(t-s) u(s) d s+f(t) \\
\text { for } t \in[0, T] \text { and } u(0)=x,
\end{gathered}
$$

where $A$ is a closed linear operator whose domain is not necessarily dense in a Banach space $X$, and $\{B(t): t \geq 0\}$ is a family of linear operators in $X$ with $D(A) \subset D(B(t))$ for $t \geq 0$ and of bounded linear operators from $Y$ into $X$. Here $Y$ is a Banach space $D(A)$ endowed with the graph norm of $A$. Roughly speaking, the integrated resolvent operator is the "integral" of the solution to the problem (VE) when the forcing term $f \equiv 0$. Our main purpose is to construct the integrated resolvent operator under the suitable conditions on $A$ and $\{B(t): t \geq 0\}$. The results obtained are applied to two Cauchy problems :

$$
\begin{gathered}
u^{\prime \prime}(t)-A u^{\prime}(t)-B u(t)=f(t) \\
\text { for } t \in[0, T], u(0)=x \text { and } u^{\prime}(0)=y \\
u^{\prime}(t)=A\left(u(t)+\int_{0}^{t} F(t-s) u(s) d s\right)+K u(t)+f(t) \\
\text { for } t \in[0, T] \text { and } u(0)=x .
\end{gathered}
$$

As illustrations of our abstract theory, two concrete examples are given.

1. Introduction. Let $X$ be a Banach space with norm $\|\cdot\|$ and denote by $B(X)$ the set of all bounded linear operators from $X$ into itself. This paper is concerned with the linear Volterra integrodifferential

Received by the editors on July 20, 1993, and in revised form on October 15, 1994.

1991 Mathematics Subject Classification. Primary 45N05, Secondary 44D05.

Key words and phrases. Integrated resolvent operator, resolvent operator, integral solution, weak solution, integrated semigroup.

Copyright (C1995 Rocky Mountain Mathematics Consortium 
equation

$$
\begin{aligned}
& u^{\prime}(t)=A u(t)+\int_{0}^{t} B(t-s) u(s) d s+f(t) \quad \text { for } t \in[0, T] \\
& u(0)=x
\end{aligned}
$$

in $X$. Here $A$ is a closed linear operator whose domain is not necessarily dense in $X$ satisfying the Hille-Yosida condition: there are real constants $M$ and $\omega$ such that

$$
\begin{aligned}
& (\omega, \infty) \subset \rho(A) \quad(\text { the resolvent set of } A) \text { and } \\
& \left\|(\lambda-A)^{-n}\right\| \leq M /(\lambda-\omega)^{n} \text { for } n=1,2, \ldots \text { and } \lambda>\omega .
\end{aligned}
$$

For convenience we introduce a Banach space $Y=D(A)$ endowed with the graph norm of $A$, i.e., $\|y\|_{Y}=\|y\|+\|A y\|$ for $y \in Y$.

$\{B(t): t \geq 0\}$ is a family of linear operators in $X$ with $D(A) \subset$ $D(B(t))$ for $t \geq 0$ and of bounded linear operators from $Y$ into $X$.

The abstract Cauchy problem

$$
\begin{aligned}
& u^{\prime}(t)=A u(t)+f(t) \quad \text { for } t \in[0, T] \\
& u(0)=x
\end{aligned}
$$

for such an operator $A$ has been recently studied by many authors (see $[\mathbf{1}, 5,12$ and 19]). Among others, Kellermann and Hieber [12] have shown that a closed linear operator $A$ in $X$ satisfying the Hille-Yosida condition (H.-Y.) generates a locally Lipschitz continuous integrated semigroup $\{U(t): t \geq 0\}$ and that if $x \in D(A), f \in W^{1,1}(0, T ; X)$ and $A x+f(0) \in \overline{D(A)}$, there exists a unique classical solution $u$ to (ACP) which is given by the variation of constants formula

$$
u(t)=\frac{d}{d t}\left(U(t) x+\int_{0}^{t} U(t-s) f(s) d s\right)
$$

for $t \in[0, T]$. Here we say that $\{U(t): t \geq 0\}$ is locally Lipschitz continuous if for $T>0$ there exists an $L_{T}>0$ such that $\| U(t)-$ $U(s) \| \leq L_{T}|t-s|$ for $s, t \in[0, T]$.

Our approach to (VE) is based on the theory of integrated semigroups, and we introduce a family $\{R(t): t \geq 0\}$ in $B(X)$ satisfying (r1)-(r4) below. 
(r1) For all $x \in X, R(\cdot) x \in C([0, \infty) ; X)$.

(r2) For all $x \in X, \int_{0} R(s) x d s \in C([0, \infty) ; Y)$.

(r3) $R(t) x-t x=A \int_{0}^{t} R(s) x d s+\int_{0}^{t} B(t-s) \int_{0}^{s} R(r) x d r d s$ for all $x \in X$ and $t \geq 0$.

(r4) $R(t) x-t x=\int_{0}^{t} R(s) A x d s+\int_{0}^{t} \int_{0}^{s} R(s-r) B(r) x d r d s$ for all $x \in D(A)$ and $t \geq 0$.

We call this family $\{R(t): t \geq 0\}$ an integrated resolvent operator and say that (VE) admits an integrated resolvent operator if it exists. If $\overline{D(A)}=X$ and $\rho(A) \neq \phi$, (VE) admits a locally Lipschitz continuous integrated resolvent operator $\{R(t): t \geq 0\}$ if and only if (VE) admits a resolvent operator $\{T(t): t \geq 0\}$. In this case, $R(t) x=\int_{0}^{t} T(s) x d s$ for $t \geq 0$ and $x \in X$ (Theorem 2.9). This is why we call a family $\{R(t): t \geq 0\}$ in $B(X)$ an integrated resolvent operator. Here we recall that a family $\{T(t): t \geq 0\}$ in $B(X)$ is a resolvent operator if

(t1) $T(0)=I$ (identity),

(t2) for all $x \in X, T(\cdot) x \in C([0, \infty) ; X)$,

(t3) for all $y \in Y, T(\cdot) y \in C^{1}([0, \infty) ; X) \cap C([0, \infty) ; Y)$,

(i) $(d / d t) T(t) y=A T(t) y+\int_{0}^{t} B(t-s) T(s) y d s$ and

(ii) $(d / d t) T(t) y=T(t) A y+\int_{0}^{t} T(t-s) B(s) y d s$ for $t \geq 0$.

Moreover if $B(t) \equiv 0$, the notion of an integrated resolvent operator coincides with that of an integrated semigroup (see [13, Theorem 3.1]). In Section 2, we investigate some properties on an integrated resolvent operator $\{R(t): t \geq 0\}$. For example, the following are proved. Let $x \in X$ and $f \in C([0, T] ; X)$, and define a function $v \in C([0, T] ; X)$ by

$$
v(t)=R(t) x+\int_{0}^{t} R(t-s) f(s) d s \quad \text { for } t \in[0, T] .
$$

If there exists a classical solution $u$ to (VE), then $v \in C^{2}([0, T] ; X)$ and $u=v^{\prime}$ (Proposition 2.3 (ii)). Conversely if $v \in C^{2}([0, T] ; X)$ and $\rho(A) \neq \phi$, then $u:=v^{\prime}$ is a classical solution to (VE) (Theorem 2.4 (ii)). Further assume that $\{R(t): t \geq 0\}$ is locally Lipschitz continuous. If $x \in D(A), f \in W^{1,1}(0, T ; X)$ and $A x+f(0) \in \overline{D(A)}$, then $v \in C^{2}([0, T] ; X)$ and so $u:=v^{\prime}$ is a classical solution to (VE) 
which satisfies

$$
\|u(t)\| \leq C\left(\|x\|+\int_{0}^{t}\|f(s)\| d s\right)
$$

and

$$
\left\|u^{\prime}(t)\right\| \leq C\left(\|A x+f(0)\|+\int_{0}^{t}\left\|B(s) x+f^{\prime}(s)\right\| d s\right)
$$

for $t \in[0, T]$, where $C$ is a constant independent of $x$ and $f$ (see Theorem 2.7). This extends the existence and uniqueness result $[\mathbf{5}$, Theorem 8.1] of classical solutions to (ACP) to the case of (VE) and the estimates (1.1) and (1.2) of a classical solution correspond to (8.4) and (8.5) in [5, Theorem 8.1], respectively. Therefore our main concern is to construct a locally Lipschitz continuous integrated resolvent operator, which will be done in Section 3. An integral solution to (VE) is also studied and it is a natural extension of that to (ACP) introduced by $\mathrm{Da}$ Prato and Sinestrari [5] (see Definition 1.1). It is proved that the notion of an integral solution coincides with that of a weak solution introduced by Grimmer and Schappacher [10] if $\overline{D(A)}=X$ and $\rho(A) \neq \phi$ (Proposition 2.8). As a consequence, we see that (VE) admits a resolvent operator if and only if (VE) has a unique weak solution for all $x \in X$ (see Theorem 2.9). This gives an improvement on [10, Theorem 3.1] where the additional assumption on $A^{*}$ and $B^{*}(t)$ (the adjoint operators of $A$ and $B(t)$, respectively) is imposed.

In Section 3 we construct a locally Lipschitz continuous integrated resolvent operator under the assumptions that a closed linear operator $A$ in $X$ satisfies the Hille-Yosida condition (H.-Y.) and that functions $B(\cdot) x$ are of strong bounded variation on each finite interval $[0, T]$ for $x \in D(A)$, which is our main result (Theorem 3.2) in this paper. The results obtained are applied to two Cauchy problems:

$$
\begin{gathered}
u^{\prime \prime}(t)-A u^{\prime}(t)-B u(t)=f(t) \quad \text { for } t \in[0, T], u(0)=x \text { and } u^{\prime}(0)=y ; \\
u^{\prime}(t)=A\left(u(t)+\int_{0}^{t} F(t-s) u(s) d s\right)+K u(t)+f(t) \\
\text { for } t \in[0, T] \text { and } u(0)=x .
\end{gathered}
$$

The last section, Section 4, is devoted to concrete examples which illustrate our abstract theory. 
We start with the definition of an integral solution and a classical solution to (VE).

Definition 1.1. Let $f \in L^{1}(0, T ; X)$ and $x \in X$. A function $u:[0, T] \rightarrow X$ is called an integral solution to $(\mathrm{VE})$ if

(i1) $u \in C([0, T] ; X)$,

(i2) $\int_{0} u(s) d s \in C([0, T] ; Y)$ and

(i3) $u(t)=x+A \int_{0}^{t} u(s) d s+\int_{0}^{t} B(t-s) \int_{0}^{s} u(r) d r d s+\int_{0}^{t} f(s) d s$ for $t \in[0, T]$.

Definition 1.2. A function $u:[0, T] \rightarrow X$ is called a classical solution to (VE) if $u \in C^{1}([0, T] ; X) \cap C([0, T] ; Y)$ and $u$ satisfies (VE).

2. Integrated resolvent operators. In this section let $A$ be a closed linear operator in $X$ and

(H0) $\{B(t): t \geq 0\}$ is assumed to be a family of linear operators in $X$ with $D(A) \subset D(B(t))$ for all $t \geq 0$ and of bounded linear operators from $Y$ into $X$ which satisfies that the functions $B(\cdot) x$ are strongly measurable on $\mathbf{R}^{+}$for $x \in D(A)$ and that there is a function $b \in L_{l o c}^{1}\left(\mathbf{R}^{+} ; \mathbf{R}^{+}\right)$such that

$$
\|B(t) x\| \leq b(t)(\|x\|+\|A x\|) \quad \text { for all } \quad x \in D(A) \text { and a.e. } t \geq 0 \text {. }
$$

Put $j_{1}(t)=t$ for $t \geq 0$.

Definition 2.1. A family $\{R(t): t \geq 0\}$ in $B(X)$ is called an integrated resolvent operator for (VE) having the following properties.

(r1) For all $x \in X, R(\cdot) x \in C([0, \infty) ; X)$.

(r2) For all $x \in X, \int_{0}^{\cdot} R(s) x d s \in C([0, \infty) ; Y)$.

(r3) $R(t) x-t x=A \int_{0}^{t} R(s) x d s+\int_{0}^{t} B(t-s) \int_{0}^{s} R(r) x d r d s$ for all $x \in X$ and $t \geq 0$.

(r4) $R(t) x-t x=\int_{0}^{t} R(s) A x d s+\int_{0}^{t} \int_{0}^{s} R(s-r) B(r) x d r d s$ for all $x \in D(A)$ and $t \geq 0$. 
Let $f \in L^{1}(0, T ; X)$. We shall use the abbreviation

$$
(R * f)(t)=\int_{0}^{t} R(t-s) f(s) d s
$$

for the convolution. Put $S(t) x=\int_{0}^{t} R(s) x d s$ for $t \geq 0$ and $x \in X$. Then by Fubini's theorem we have

$$
\begin{aligned}
\int_{0}^{t}(R * f)(s) d s & =\int_{0}^{t} \int_{0}^{s} R(s-r) f(r) d r d s \\
& =\int_{0}^{t} \int_{r}^{t} R(s-r) f(r) d s d r \\
& =\int_{0}^{t} \int_{0}^{t-r} R(s) f(r) d s d r \\
& =(S * f)(t) .
\end{aligned}
$$

In this section we assume that (VE) admits an integrated resolvent operator $\{R(t): t \geq 0\}$ and investigate its properties.

Proposition 2.1. Suppose that $u \in C([0, \infty) ; X), \int_{0}^{*} u(s) d s \in$ $C([0, \infty) ; Y)$ and

$$
u(t)=A \int_{0}^{t} u(s) d s+\int_{0}^{t} B(t-s) \int_{0}^{s} u(r) d r d s
$$

for all $t \geq 0$. Then $u(t)=0$ for $t \geq 0$.

Proof. By (r4) note that

$$
\frac{d}{d t} R(t) x-x=R(t) A x+(R * B(\cdot) x)(t)
$$

for $x \in D(A)$ and $t \geq 0$.

Put $w(t)=\int_{0}^{t} u(s) d s$ for $t \geq 0$. Convolving both sides of (2.3) with $R(t)$ from the left side, using (2.4) and integrating by parts we obtain

$$
\begin{aligned}
R * u & =R * A w+R * B * w \\
& =R^{\prime} * w-1 * w \\
& =R * u-1 * w,
\end{aligned}
$$


and so $1 * w=0$, i.e. $\int_{0}^{t} \int_{0}^{s} u(r) d r d s=\int_{0}^{t} w(s) d s=0$. Hence $u(t)=0$ for $t \geq 0$.

Corollary 2.2. (VE) admits at most one integrated resolvent operator.

Next we study the relationship between solutions to (VE) and the variation of constants formula (2.5) below which is given in terms of integrated resolvent operators.

Let $x \in X$ and $f \in L^{1}(0, T ; X)$. Consider a function $v \in C([0, T] ; X)$ given by

$$
v(t)=R(t) x+\int_{0}^{t} R(t-s) f(s) d s \quad \text { for } t \in[0, T] .
$$

Proposition 2.3. The following hold.

(i) If there exists an integral solution $u$ to (VE), then $v \in$ $C^{1}([0, T] ; X)$ and $u=v^{\prime}$.

(ii) If there exists a classical solution $u$ to (VE), then $v \in$ $C^{2}([0, T] ; X)$ and $u=v^{\prime}$.

Proof. We shall prove (i). Let $u$ be an integral solution to (VE). Put $w(t)=\int_{0}^{t} u(s) d s$ and $g(t)=\int_{0}^{t} f(s) d s$ for $t \in[0, T]$. Convolving both sides of the equality $u=x+A w+B * w+g$ with $R(t)$ from the left side we have

$$
\begin{aligned}
(R * u)(t) & =S(t) x+(R * A w)(t)+(R * B * w)(t)+(R * g)(t) \\
& =S(t) x+(R * u)(t)-(1 * w)(t)+(R * g)(t),
\end{aligned}
$$

i.e. $\int_{0}^{t} \int_{0}^{s} u(r) d r d s=\int_{0}^{t} w(s) d s=S(t) x+(R * g)(t)=\int_{0}^{t} v(s) d s$. Differentiating this yields $v(t)=\int_{0}^{t} u(s) d s$, which means $v \in C^{1}([0, T] ; X)$ and $u=v^{\prime}$. The assertion (ii) is also similarly proved.

Conversely, we have

Theorem 2.4. Suppose $\rho(A) \neq \phi$. Then the following hold. 
(i) If $v \in C^{1}([0, T] ; X)$, then $u:=v^{\prime}$ is an integral solution to (VE).

(ii) Assume $f \in C([0, T] ; X)$. If $v \in C^{2}([0, T] ; X)$, then $u:=v^{\prime}$ is a classical solution to (VE).

The proof of Theorem 2.4 is based on the following lemma.

Lemma 2.5. $\int_{0} v(s) d s \in C([0, T] ; Y)$ and

$$
\begin{aligned}
A \int_{0}^{t} v(s) d s= & v(t)-t x-\int_{0}^{t} \int_{0}^{s} f(r) d r d s \\
& -\int_{0}^{t} B(t-s) \int_{0}^{s} v(r) d r d s
\end{aligned}
$$

for $t \in[0, T]$.

Proof. By (r2) and (r3) we have $S(t-r) f(r) \in D(A)$ and

$$
\begin{aligned}
A S(t-r) f(r)= & R(t-r) f(r)-(t-r) f(r) \\
& -\int_{0}^{t-r} B(t-r-s) S(s) f(r) d s \in L^{1}(0, T ; X) .
\end{aligned}
$$

From the closedness of $A$ we deduce $S * f \in D(A)$ and $A(S * f)=$ $A S * f \in C([0, T] ; X)$. In view of $(\mathrm{r} 2)$ and $(2.2)$ we have $\int_{0}^{*} v(s) d s \in$ $C([0, T] ; Y)$. Convolving both sides of $(\mathrm{r} 3)$ with $f(t)$ from the right side and using (2.2) we find

$$
\begin{aligned}
(R * f)(t)-\left(j_{1} * f\right)(t) & =(A S * f)(t)+(B * S * f)(t) \\
& =A \int_{0}^{t}(R * f)(s) d s+\left(B * \int_{0}(R * f)(r) d r\right)(t) .
\end{aligned}
$$

Adding the above equality to (r3) we have the desired equality (2.6).

口

Proof of Theorem 2.4. Let $\lambda_{0} \in \rho(A)$ and define an operator $K(t) \in B(X)$ by $K(t)=-B(t)\left(\lambda_{0}-A\right)^{-1}$ for $t \in[0, T]$. Let $L(t)$ denote the resolvent kernel for $K(t)$, i.e. $L(\cdot) z \in L_{\text {loc }}^{1}\left(\mathbf{R}^{+} ; X\right)$ and $L(t) z=K(t) z-(L * K)(t) z$ for almost every $t \in[0, T]$ and 
$z \in X$. Set $\int_{0}^{t} v(s) d s=w(t)$ for $t \in[0, T]$. Lemma 2.5 implies that $w(\cdot) \in C([0, T] ; Y)$ and $(A w)(t)=v(t)-t x-\int_{0}^{t} \int_{0}^{s} f(r) d r d s-(B * w)(t)$ for $t \in[0, T]$. Since $B(t) y=K(t) A y-\lambda_{0} K(t) y$ for $y \in D(A)$, we have

$$
\begin{aligned}
(B * w)(t) & =((K-L * K) * A w)(t)+(L * K A * w)(t)-\lambda_{0}(K * w)(t) \\
& =(L * A w)(t)+\left(L *\left(B+\lambda_{0} K\right) * w\right)(t)-\lambda_{0}(K * w)(t) \\
& =(L *(A w+B * w))(t)+\lambda_{0}((L * K-K) * w)(t) \\
& =(L * z)(t)-\lambda_{0}(L * w)(t)
\end{aligned}
$$

where $z(t)=v(t)-t x-\int_{0}^{t} \int_{0}^{s} f(r) d r d s$ for $t \in[0, T]$.

First we shall prove (i). Assume $v \in C^{1}([0, T] ; X)$. In view of $(2.7)$ we find that $(B * w)(t)$ is continuously differentiable in $t$. Since $A$ is closed, we may differentiate $(2.6)$ to obtain that $v(\cdot) \in C([0, T] ; Y)$ and

$$
A v(t)=v^{\prime}(t)-x-\int_{0}^{t} f(s) d s-(B * v)(t) \quad \text { for } t \in[0, T] .
$$

Noting $v(0)=0$, we see that $u:=v^{\prime}$ is an integral solution to (VE).

Next assume $f \in C([0, T] ; X)$ and $v \in C^{2}([0, T] ; X)$. From (2.7) and $(2.8)$ it follows that $v^{\prime}(0)=x$ and $(B * v)(\cdot)=\left(L * z^{\prime}\right)(\cdot)-$ $\lambda_{0}(L * v)(\cdot) \in C^{1}([0, T] ; X)$. By the closedness of $A$ again we deduce that $v^{\prime} \in C([0, T] ; Y)$ and the differentiation of $(2.8)$ gives $A v^{\prime}(t)=$ $v^{\prime \prime}(t)-f(t)-\left(B * v^{\prime}\right)(t)$ for $t \in[0, T]$. This means that $u:=v^{\prime}$ is a classical solution to (VE), which proves (ii).

The following lemma for a locally Lipschitz continuous family is simple but important for our discussion.

Lemma 2.6. Let a family $\{U(t): t \geq 0\}$ in $B(X)$ be locally Lipschitz continuous with $U(0)=0$. Then we have the following.

(i) If $f \in L^{1}(0, T ; X)$, then $\int_{0} U(\cdot-s) f(s) d s \in C^{1}([0, T] ; X)$. Putting $u(t):=(d / d t) \int_{0}^{t} U(t-s) f(s) d s$, we have

$$
\|u(t)\| \leq C_{T} \int_{0}^{t}\|f(s)\| d s \quad \text { for } t \in[0, T]
$$


where $C_{T}$ is the Lipschitz constant of $\{U(t): t \in[0, T]\}$. Moreover, if $\|f(t)\| \leq K$ for $t \in[0, T]$,

$$
\|u(t+s)-u(t)\| \leq K C_{T} s+C_{T} \int_{0}^{t}\|f(s+r)-f(r)\| d r
$$

for $s, t, t+s \in[0, T]$.

(ii) If a function $f:[0, T] \rightarrow X$ is of strong bounded variation, the function $u(\cdot)$ defined in (i) is Lipschitz continuous on $[0, T]$.

Proof. The first assertion can be proved in the same manner as in [12, pp. 167-168]. Since $\|U(t)\| \leq C_{T} t$ for $t \in[0, T]$ by the assumption, integration by parts yields

$$
\begin{aligned}
\left\|\frac{1}{h} \int_{t}^{t+h} U(t+h-s) f(s) d s\right\| & \leq \frac{C_{T}}{h} \int_{t}^{t+h}(t+h-s)\|f(s)\| d s \\
& =\frac{C_{T}}{h} \int_{t}^{t+h} \int_{t}^{s}\|f(r)\| d r d s \rightarrow 0 \\
& \text { as } h \rightarrow 0+.
\end{aligned}
$$

Therefore

$$
\begin{aligned}
u(t) & =\lim _{h \rightarrow 0+} \frac{1}{h}\left(\int_{0}^{t+h} U(t+h-s) f(s) d s-\int_{0}^{t} U(t-s) f(s) d s\right) \\
& =\lim _{h \rightarrow 0+} \frac{1}{h} \int_{0}^{t}(U(t+h-s)-U(t-s)) f(s) d s .
\end{aligned}
$$

From this and the Lipschitz continuity of $\{U(t): t \in[0, T]\}$ the estimate (2.9) follows. It is easy to see that the inequality (2.10) holds by virtue of the equality (2.11).

Finally we shall prove (ii).

Put $V_{f}(t):=\sup \left\{\sum_{i=1}^{n}\left\|f\left(t_{i}\right)-f\left(t_{i-1}\right)\right\|: 0 \leq t_{0}<t_{1}<\cdots<t_{n} \leq\right.$ $t ; n \geq 1\}$ for $t \in[0, T]$. Then $V_{f}(t)$ is a nondecreasing function and

$$
\|f(t)-f(s)\| \leq V_{f}(t)-V_{f}(s) \text { for } 0 \leq s \leq t \leq T
$$


Then we have for $s, t, t+s \in[0, T]$

$$
\begin{aligned}
\int_{0}^{t}\|f(s+r)-f(r)\| d r & \leq \int_{0}^{t} V_{f}(s+r) d r-\int_{0}^{t} V_{f}(r) d r \\
& =\int_{s}^{t+s} V_{f}(r) d r-\int_{0}^{t} V_{f}(r) d r \\
& =\int_{t}^{t+s} V_{f}(r) d r-\int_{0}^{s} V_{f}(r) d r \\
& \leq s V_{f}(T),
\end{aligned}
$$

which implies with (2.10) the desired conclusion.

Theorem 2.7. Suppose that an integrated resolvent operator $\{R(t)$ : $t \geq 0\}$ is locally Lipschitz continuous and that $\rho(A) \neq \phi$. Then the following hold.

(i) If $x \in \overline{D(A)}$ and $f \in L^{1}(0, T ; X)$, then there exists a unique integral solution $u(\cdot)$ to $(\mathrm{VE})$. Moreover we have

$$
\|u(t)\| \leq C\left(\|x\|+\int_{0}^{t}\|f(s)\| d s\right) \quad \text { for } t \in[0, T],
$$

where $C$ is a constant independent of $x$ and $f$.

(ii) If $x \in D(A), f \in W^{1,1}(0, T ; X)$ and $A x+f(0) \in \overline{D(A)}$, then there exists a unique classical solution $u(\cdot)$ to $(\mathrm{VE})$. Moreover we have

$$
\left\|u^{\prime}(t)\right\| \leq C\left(\|A x+f(0)\|+\int_{0}^{t}\left\|B(s) x+f^{\prime}(s)\right\| d s\right) \quad \text { for } t \in[0, T] .
$$

Proof. First we shall show (i). By Proposition 2.3 (i) and Theorem 2.4 (i) it suffices to prove $v \in C^{1}([0, T] ; X)$. Since $R(\cdot) y \in C^{1}([0, T] ; X)$ for $y \in D(A)$ by $(\mathrm{r} 4)$, the Lipschitz continuity of $\{R(t): t \in[0, T]\}$ implies $R(\cdot) x \in C^{1}([0, T] ; X)$. Since $R(0)=0$ by (r3), from Lemma 2.6 (i) we see that the function $t \mapsto \int_{0}^{t} R(t-s) f(s) d s$ belongs to $C^{1}$. Therefore $v \in C^{1}([0, T] ; X)$. Because $u(t):=v^{\prime}(t)=R^{\prime}(t) x+(d / d t) \int_{0}^{t} R(t-$ $s) f(s) d s$ for $t \in[0, T]$, we have the estimate (2.13) of an integral 
solution $u(\cdot)$ by the Lipschitz continuity of $\{R(t): t \in[0, T]\}$ and the inequality (2.9).

Next we shall prove (ii). We have only to prove $v \in C^{2}([0, T] ; X)$ by Proposition 2.3 (ii) and Theorem 2.4 (ii). By (r4) we have

$$
\begin{aligned}
v^{\prime}(t)= & x+R(t) A x+\int_{0}^{t} R(t-s) B(s) x d s \\
& +R(t) f(0)+\int_{0}^{t} R(t-s) f^{\prime}(s) d s
\end{aligned}
$$

for $t \in[0, T]$. Since $A x+f(0) \in \overline{D(A)}, B(\cdot) x \in L^{1}(0, T ; X)$ by $(2.1)$ and $f^{\prime}(\cdot) \in L^{1}(0, T ; X)$, the above consideration yields $v \in C^{2}([0, T] ; X)$. Since the equality

$$
\begin{aligned}
u^{\prime}(t)=v^{\prime \prime}(t)= & R^{\prime}(t)(A x+f(0)) \\
& +\frac{d}{d t} \int_{0}^{t} R(t-s)\left(B(s) x+f^{\prime}(s)\right) d s
\end{aligned}
$$

holds, the Lipschitz continuity of $\{R(t): t \in[0, T]\}$ and the inequality (2.9) imply the estimate (2.14) of the derivative of a classical solution $u(\cdot)$.

Throughout the rest of this section we suppose that $\overline{D(A)}=X$ and $\rho(A) \neq \phi$.

We will show that an integral solution to (VE) coincides with a weak solution introduced by Grimmer and Schappacher [10].

In what follows, in addition to the condition (H0) we shall impose the following assumption on the adjoint operators $A^{*}$ and $B^{*}(t)$ of $A$ and $B(t)$, respectively.

(H1) $D\left(A^{*}\right) \subset D\left(B^{*}(t)\right)$ for $t \geq 0$ and functions $B^{*}(\cdot) x^{*}$ are strongly measurable on $\mathbf{R}^{+}$for $x^{*} \in D\left(A^{*}\right)$. Further,

$$
\left\|B^{*}(t) x^{*}\right\| \leq b(t)\left(\left\|A^{*} x^{*}\right\|+\left\|x^{*}\right\|\right)
$$

for $x^{*} \in D\left(A^{*}\right)$ and almost every $t \geq 0$.

According to Grimmer and Schappacher [10], we recall the definition of a weak solution to ( $\mathrm{VE})$. 
Definition 2.2. A function $u:[0, T] \rightarrow X$ is called a weak solution to (VE) if

(w1) $u \in C([0, T] ; X)$,

(w2) for all $v \in D\left(A^{*}\right)$, the map $t \mapsto\langle u(t), v\rangle$ is absolutely continuous on $[0, T]$,

$$
\frac{d}{d t}\langle u(t), v\rangle=\left\langle u(t), A^{*} v\right\rangle+\int_{0}^{t}\left\langle u(s), B^{*}(t-s) v\right\rangle d s+\langle f(t), v\rangle
$$

for almost every $t \in[0, T]$ and

(w3) $u(0)=x$.

The relation between an integral solution and a weak solution is given by

Proposition 2.8. Let $x \in X$. A function $u:[0, T] \rightarrow X$ is an integral solution to (VE) if and only if $u$ is a weak solution to (VE).

Proof. Suppose that $u:[0, T] \rightarrow X$ is an integral solution to (VE). Let $v \in D\left(A^{*}\right)$. Since

$$
\begin{aligned}
\left\langle A \int_{0}^{t} u(s) d s, v\right\rangle & =\left\langle\int_{0}^{t} u(s) d s, A^{*} v\right\rangle \\
& =\int_{0}^{t}\left\langle u(s), A^{*} v\right\rangle d s
\end{aligned}
$$

and

$$
\begin{aligned}
\left\langle\int_{0}^{t} B(t-s) \int_{0}^{s} u(r) d r d s, v\right\rangle & =\int_{0}^{t}\left\langle B(t-s) \int_{0}^{s} u(r) d r, v\right\rangle d s \\
& =\int_{0}^{t}\left\langle\int_{0}^{t-s} u(r) d r, B^{*}(s) v\right\rangle d s
\end{aligned}
$$

we have

$$
\begin{aligned}
\langle u(t), v\rangle= & \langle x, v\rangle+\int_{0}^{t}\left\langle u(s), A^{*} v\right\rangle d s+\int_{0}^{t}\left\langle\int_{0}^{t-s} u(r) d r, B^{*}(s) v\right\rangle d s \\
& +\int_{0}^{t}\langle f(s), v\rangle d s
\end{aligned}
$$


for $t \in[0, T]$, which implies that the map $t \mapsto\langle u(t), v\rangle$ is absolutely continuous on $[0, T]$. Differentiating this, we obtain

$$
\begin{aligned}
\frac{d}{d t}\langle u(t), v\rangle= & \left\langle u(t), A^{*} v\right\rangle+\int_{0}^{t}\left\langle u(t-s), B^{*}(s) v\right\rangle d s+\langle f(t), v\rangle \\
= & \left\langle u(t), A^{*} v\right\rangle+\int_{0}^{t}\left\langle u(s), B^{*}(t-s) v\right\rangle d s \\
& +\langle f(t), v\rangle \text { for a.e. } t \in[0, T]
\end{aligned}
$$

This means that $u$ is a weak solution to (VE).

Without loss of generality we may assume $0 \in \rho\left(A^{*}\right)$. Conversely, let $u:[0, T] \rightarrow X$ be a weak solution to (VE). Integrating the equality (w2), we have

$$
\begin{aligned}
\langle u(t), v\rangle-\langle x, v\rangle= & \int_{0}^{t}\left\langle u(s), A^{*} v\right\rangle d s \\
& +\int_{0}^{t} \int_{0}^{s}\left\langle u(r), B^{*}(s-r) v\right\rangle d r d s+\int_{0}^{t}\langle f(s), v\rangle d s
\end{aligned}
$$

for $t \in[0, T]$. By Fubini's theorem and integration by parts we get

$$
\begin{aligned}
\int_{0}^{t} \int_{0}^{s}\left\langle u(r), B^{*}(s-r) v\right\rangle d r d s & =\int_{0}^{t} \int_{r}^{t}\left\langle u(r), B^{*}(s-r) v\right\rangle d s d r \\
& =\int_{0}^{t}\left\langle u(r), \int_{r}^{t} B^{*}(s-r) v d s\right\rangle d r \\
& =\int_{0}^{t}\left\langle u(r), \int_{0}^{t-r} B^{*}(s) v d s\right\rangle d r \\
& =\int_{0}^{t}\left\langle\int_{0}^{r} u(s) d s, B^{*}(t-r) v\right\rangle d r
\end{aligned}
$$

Moreover, we have

$$
\begin{aligned}
\int_{0}^{t}\left\langle\int_{0}^{s} u(r) d r, B^{*}\right. & (t-s) v\rangle d s \\
& =\left\langle\int_{0}^{t}\left(B^{*}(t-s)\left(A^{*}\right)^{-1}\right)^{*} \int_{0}^{s} u(r) d r d s, A^{*} v\right\rangle
\end{aligned}
$$


for $t \in[0, T]$. By [10, Lemma 4.2] and its proof we see that the operator $A^{-1} B(t)$ has a bounded extension, $\widetilde{A^{-1} B(t)}$ to $X$ for almost every $t \geq 0$ such that $\widetilde{A^{-1} B(t)} z$ is locally Bochner integrable in $t$ for each $z \in X$ and that $\widetilde{A^{-1} B(t)} z=\left(B^{*}(t)\left(A^{*}\right)^{-1}\right)^{*} z$ for almost every $t \geq 0$ and $z \in X$. Therefore we have

$$
\begin{aligned}
\langle u(t) & \left.-x-\int_{0}^{t} f(s) d s, v\right\rangle \\
& =\left\langle\int_{0}^{t} u(s) d s+\int_{0}^{t}\left(B^{*}(t-s)\left(A^{*}\right)^{-1}\right)^{*} \cdot \int_{0}^{s} u(r) d r d s, A^{*} v\right\rangle \\
& =\left\langle\int_{0}^{t} u(s) d s+\int_{0}^{t} \widetilde{A^{-1} B}(t-s) \cdot \int_{0}^{s} u(r) d r d s, A^{*} v\right\rangle
\end{aligned}
$$

for $t \in[0, T]$. Putting $y(t)=\int_{0}^{t} u(s) d s+\int_{0}^{t} \widetilde{A^{-1} B}(t-s) \int_{0}^{s} u(r) d r d s$ for $t \in[0, T]$, by [3, Lemma] we find that $y(t) \in D(A)$ and $A y(t)=$ $u(t)-x-\int_{0}^{t} f(s) d s$ is continuous in $t \in[0, T]$. Now the integral equation

$$
z(t)=y(t)-\int_{0}^{t} \widetilde{A^{-1} B}(t-s) z(s) d s
$$

has a unique solution $z_{1}(\cdot) \in C([0, T] ; X)$ because $\widetilde{A^{-1} B(\cdot)} z \in$ $L_{\text {loc }}^{1}\left(\mathbf{R}^{+} ; X\right)$ for each $z \in X$ as noted above. Therefore, we have $\int_{0}^{t} u(s) d s=z_{1}(t)$ for $t \in[0, T]$. However, $\widetilde{A^{-1} B(t)}$ maps $Y$ into $Y$, and for $y \in Y$ and almost every $t \geq 0$,

$$
\left\|A^{-1} B(t) y\right\|_{Y}=\|B(t) y\|+\left\|A^{-1} B(t) y\right\| \leq\left(1+\left\|A^{-1}\right\|\right) b(t)\|y\|_{Y} .
$$

Thus, $A^{-1} B(t) \in B(Y)$ for almost every $t \geq 0$ and $A^{-1} B(t) y$ is locally Bochner integrable in $Y$ for each $y \in Y$. Noting the fact that $y(\cdot) \in C([0, T] ; Y)$, we see that $(2.15)$ has a unique solution $z_{2}(\cdot) \in C([0, T] ; Y)$. Because of $z_{2}(\cdot) \in C([0, T] ; X)$, by the uniqueness of solutions to $(2.15)$ in $C([0, T] ; X)$ we have $\int_{0}^{\cdot} u(s) d s=z_{1}(\cdot)=z_{2}(\cdot) \in$ $C([0, T] ; Y)$. So we have

$$
\int_{0}^{t}\left\langle\int_{0}^{s} u(r) d r, B^{*}(t-s) v\right\rangle d s=\left\langle\int_{0}^{t} B(t-s) \int_{0}^{s} u(r) d r d s, v\right\rangle
$$


and

$$
\int_{0}^{t}\left\langle u(s), A^{*} v\right\rangle d s=\left\langle\int_{0}^{t} u(s) d s, A^{*} v\right\rangle=\left\langle A \int_{0}^{t} u(s) d s, v\right\rangle
$$

for $v \in D\left(A^{*}\right)$ and $t \in[0, T]$. By [3, Lemma] again, we find that $u$ is an integral solution to (VE).

Theorem 2.9. Suppose that $\overline{D(A)}=X$ and $\rho(A) \neq \phi$. Let $f \in L_{\text {loc }}^{1}\left(\mathbf{R}^{+} ; X\right)$. The following statements are equivalent:

(i) (VE) admits a locally Lipschitz continuous integrated resolvent operator $\{R(t): t \geq 0\}$.

(ii) (VE) admits a resolvent operator $\{T(t): t \geq 0\}$.

(iii) For all $x \in X$ there exists a unique integral solution $u$ to (VE).

(iv) For all $x \in X$ there exists a unique weak solution $u$ to (VE). In this case,

$$
\begin{aligned}
R(t) x & =\int_{0}^{t} T(s) x d s \\
u(t) & =\frac{d}{d t}\left(R(t) x+\int_{0}^{t} R(t-s) f(s) d s\right) \\
& =T(t) x+\int_{0}^{t} T(t-s) f(s) d s \quad \text { for } t \geq 0 \text { and } x \in X .
\end{aligned}
$$

Proof. By Theorem 2.7 (i) we have the implication (i) $\Rightarrow$ (iii). Since Proposition 2.8 shows the equivalence (iii) $\Leftrightarrow$ (iv), it remains to prove that implications (iii) $\Rightarrow$ (ii) and (ii) $\Rightarrow$ (i).

To show that (iii) implies (ii), let $u(\cdot ; x)$ be a unique integral solution to (VE) for each $x \in X$. For $t \geq 0$ define an operator $T(t)$ on $X$ to $X$ by $T(t) x=u(t ; x)-u(t ; 0)$ for $x \in X$. Then from the definition of integral solutions to (VE) it follows that

$$
T(t) x=x+A \int_{0}^{t} T(s) x d s+\int_{0}^{t} B(t-s) \int_{0}^{s} T(r) x d r d s
$$

for $t \geq 0$ and $x \in X$. Clearly, $T(0)=I$ and $T(\cdot) x \in C([0, \infty) ; X)$ for $x \in X$. Then by the uniqueness of integral solutions we see that each $T(t)$ is a linear operator on $X$. 
Define an $F$-space ( in the sense of Dunford-Schwartz [7]) by

$$
\mathcal{X}=\left\{u \in C([0, \infty) ; X): \int_{0} u(s) d s \in C([0, \infty) ; Y)\right\}
$$

with the quasi-norm defined by

$$
\||v|\|=\sum_{k=1}^{\infty} \frac{1}{2^{k}} \cdot \frac{\|v\|_{k}}{1+\|v\|_{k}} \quad \text { for } v \in \mathcal{X},
$$

where $\|v\|_{k}=\max _{0 \leq t \leq k} \max \left(\|v(t)\|,\left\|\int_{0}^{t} v(s) d s\right\|_{Y}\right)$ for $k=1,2,3, \ldots$ (note that $\lim _{n \rightarrow \infty}\left|\left\|v_{n}|\||=0\right.\right.$ if and only if $\left.\lim _{n \rightarrow \infty}\right\| v_{n} \|_{k}=0$ for all $k$ ). We define the linear operator $\mathcal{T}: X \rightarrow \mathcal{X}$ by $\mathcal{T} x=T(\cdot) x$ for $x \in X$. To prove that $\mathcal{T}$ is closed, let $\left\|x_{n}-x\right\| \rightarrow 0$ and $\left\|\mid \mathcal{T} x_{n}-y\right\| \| 0$ as $n \rightarrow \infty$.

By the above remark we have $\lim _{n \rightarrow \infty}\left(\max _{0 \leq t \leq k}\left\|T(t) x_{n}-y(t)\right\|\right)=0$ and $\lim _{n \rightarrow \infty}\left(\max _{0 \leq t \leq k}\left\|\int_{0}^{t} T(s) x_{n} d s-\int_{0}^{t} y(s) d s\right\|_{Y}\right)=0$ for all $k$. Passing to the limit as $n \rightarrow \infty$ in the equality

$$
T(t) x_{n}=x_{n}+A \int_{0}^{t} T(s) x_{n} d s+\int_{0}^{t} B(t-s) \int_{0}^{s} T(r) x_{n} d r d s
$$

we have

$$
y(t)=x+A \int_{0}^{t} y(s) d s+\int_{0}^{t} B(t-s) \int_{0}^{s} y(r) d r d s
$$

for $t \geq 0$, which means that $y(\cdot)+u(\cdot ; 0)$ is an integral solution to (VE). By the uniqueness of integral solutions we have $y(\cdot)+u(\cdot ; 0)=$ $u(\cdot ; x)$, i.e., $y(\cdot)=T(\cdot) x$ and hence $y=\mathcal{T} x$. From the closed graph theorem (see [7, Ch. II, Theorem 2.2.4]) we see that $\mathcal{T}$ is a continuous operator from $X$ into $\mathcal{X}$ : hence $\lim _{n \rightarrow \infty}\left\|x_{n}-x\right\|=0$ implies $\lim _{n \rightarrow \infty}\left(\max _{0 \leq t \leq k}\left\|T(t) x_{n}-T(t) x\right\|\right)=0$ for every $k$. This shows that each $T(t): X \rightarrow X$ is continuous, that is, $T(t) \in B(X)$.

To prove (t3), let $y \in Y$ and $z(t)=y+\int_{0}^{t} T(s) A y d s+\int_{0}^{t} \int_{0}^{s} T(s-$ $r) B(r) y d r d s$ for $t \geq 0$. Since (2.16) holds, we see by the same argument as in the proof of Lemma 2.5 that $\int_{0} w(s) d s \in C([0, \infty) ; Y)$ and

$$
w(t)=A \int_{0}^{t} w(s) d s+\int_{0}^{t} B(t-s) \int_{0}^{s} w(r) d r d s+\int_{0}^{t} g(s) d s
$$


for $w(t):=(T * g)(t)$ and $g \in L_{\text {loc }}^{1}\left(\mathbf{R}^{+} ; X\right)$. Since $B(\cdot) y \in L_{\text {loc }}^{1}\left(\mathbf{R}^{+} ; X\right)$, we have $z(\cdot) \in C([0, \infty) ; Y)$. By the above equation and $z(t)-y=$ $\int_{0}^{t} T(t-s)\left(A y+\int_{0}^{s} B(r) y d r\right) d s$, from the closedness of $A$ we deduce $\int_{0}(z(s)-y) d s \in C([0, \infty) ; Y)$ and

$$
\begin{aligned}
z(t)-y= & A \int_{0}^{t}(z(s)-y) d s+\int_{0}^{t} B(t-s) \int_{0}^{s}(z(r)-y) d r d s \\
& +\int_{0}^{t}\left(A y+\int_{0}^{s} B(r) y d r\right) d s \\
= & A \int_{0}^{t} z(s) d s+\int_{0}^{t} B(t-s) \int_{0}^{s} z(r) d r d s
\end{aligned}
$$

for $t \geq 0$. Hence the uniqueness of integral solutions follows

$$
\begin{aligned}
T(t) y=z(t)= & y+\int_{0}^{t} T(s) A y d s \\
& +\int_{0}^{t} \int_{0}^{s} T(s-r) B(r) y d r d s
\end{aligned}
$$

which implies $T(\cdot) y \in C^{1}([0, \infty) ; X) \cap C([0, \infty) ; Y)$ and $(d / d t) T(t) y=$ $T(t) A y+\int_{0}^{t} T(t-s) B(s) y d s$ for $t \geq 0$, that is (t3)(ii) holds. Since $T(\cdot) y \in C^{1}([0, \infty) ; X) \cap C([0, \infty) ; Y)$ and $A$ is closed, the differentiation of (2.16) with $x$ replaced by $y \in Y$ in $t \geq 0$ gives (t3)(i). Therefore (VE) admits a resolvent operator $\{T(t): t \geq 0\}$.

Finally we show that (ii) implies (i). Suppose that (VE) admits a resolvent operator $\{T(t): t \geq 0\}$. Define an operator $R(t)$ in $B(X)$ by $R(t) x=\int_{0}^{t} T(s) x d s$ for $t \geq 0$ and $x \in X$. Clearly, $\{R(t): t \geq 0\}$ is locally Lipschitz continuous.

Let $y \in D(A)$. Integration of (t3)(i) and the closedness of $A$ yield $\int_{0}^{\cdot} T(s) y d s \in C([0, \infty) ; Y)$ and

$$
T(t) y-y=A \int_{0}^{t} T(s) y d s+\int_{0}^{t} B(t-s) \int_{0}^{s} T(r) y d r d s
$$

for $t \geq 0$. Then from (H0) and the above equality it follows that

$$
\begin{aligned}
\|R(t) y\|_{Y} & =\left\|\int_{0}^{t} T(s) y d s\right\|+\left\|A \int_{0}^{t} T(s) y d s\right\| \\
& \leq\left\|\int_{0}^{t} T(s) y d s\right\|+\|T(t) y-y\|+\int_{0}^{t} b(t-s)\|R(s) y\|_{Y} d s
\end{aligned}
$$


and hence Gronwall's inequality gives

$$
\begin{aligned}
\|R(t)\|_{Y} \leq & \left\|\int_{0}^{t} T(s) y d s\right\|+\|T(t) y-y\| \\
& +\int_{0}^{t} r(t-s)\left\{\left\|\int_{0}^{s} T(r) y d r\right\|+\|T(s) y-y\|\right\} d s
\end{aligned}
$$

where $r(t)=b(t)+(r * b)(t)$ for almost every $t \geq 0$, i.e., $r$ is a resolvent kernel of $b$. Therefore we have

$$
\|A R(t) y\| \leq\|T(t) y-y\|+\int_{0}^{t} r(t-s)(\|R(s) y\|+\|T(s) y-y\|) d s
$$

for $t \geq 0$. This shows that $A R(t)$ has a bounded extension to all of $X$ and the closedness of $A$ follows $R(t) x \in D(A)$ for all $t \geq 0$ and $x \in X$. Since $D(A)$ is dense in $X$, for $x \in X$ there exists a sequence $\left\{x_{n}\right\} \subset D(A)$ such that $\lim _{n \rightarrow \infty} x_{n}=x . A R(\cdot) x_{n} \in C([0, \infty) ; X)$ for $n \geq 1$ and in view of (2.18), we see that $\lim _{n \rightarrow \infty} A R(t) x_{n}=A R(t) x$ uniformly on any bounded subinterval. Hence $A R(\cdot) x \in C([0, \infty) ; X)$. Therefore from the closedness of $A$ we deduce (r2). Combining this fact with (2.17), by density argument we find

$$
T(t) x-x=A \int_{0}^{t} T(s) x d s+\int_{0}^{t} B(t-s) \int_{0}^{s} T(r) x d r d s
$$

for $t \geq 0$. Integrating this we have (r3) from the closedness of $A$. The property (r4) can be obtained by integrating the equation (t3) (ii) twice.

Remark. The equivalence (ii) $\Leftrightarrow$ (iv) in Theorem 2.9 improves $[\mathbf{1 0}$, Theorem 3.1] where the additional assumption on $A^{*}$ and $B^{*}(t)$ ([10, (H4)]) is imposed.

3. Main results. First we prepare some results on locally Lipschitz continuous integrated semigroups.

We recall that a closed linear operator $A$ in $X$ satisfies the HilleYosida condition (H.-Y.) if and only if $A$ is the generator of a locally Lipschitz continuous integrated semigroup $\{S(t): t \geq 0\}$ on $X$ such that

$$
\|S(t+h)-S(t)\| \leq M h e^{\omega(t+h)} \quad \text { for } t, h \geq 0
$$


(see [1, Theorem 4.1] and [12, Theorem 2.4]). The following can be obtained by using Lemmas 2.5 and 2.6. Note that an integrated resolvent operator coincides with an integrated semigroup if $B(t) \equiv 0$ (see [13, Theorem 3.1]).

Lemma 3.1. Suppose that a closed linear operator $A$ in $X$ satisfies the Hille-Yosida condition (H.-Y.). Let $\{S(t): t \geq 0\}$ be a locally Lipschitz continuous integrated semigroup on $X$ generated by $A$ and let $f \in L_{\text {loc }}^{1}\left(\mathbf{R}^{+} ; X\right)$. Then we have

$$
\int_{0} S(\cdot-s) f(s) d s \in C^{1}([0, \infty) ; X) \cap C([0, \infty) ; Y),
$$

$$
\frac{d}{d t} \int_{0}^{t} S(t-s) f(s) d s=A \int_{0}^{t} S(t-s) f(s) d s+\int_{0}^{t} f(s) d s
$$

and

$$
\left\|\frac{d}{d t} \int_{0}^{t} S(t-s) f(s) d s\right\| \leq M \int_{0}^{t} e^{\omega(t-s)}\|f(s)\| d s
$$

for $t \geq 0$.

We assume that

(H2) $\{B(t): t \geq 0\}$ is a family of linear operators in $X$ with $D(A) \subset D(B(t))$ for all $t \geq 0$ and of bounded linear operators from $Y$ into $X$ satisfying the condition that the functions $B(\cdot) x$ are of strong bounded variation on each finite interval $[0, T]$ for $x \in D(A)$.

Note that (H2) implies (H0) since a function of strong bounded variation is strongly measurable and also by the uniform boundedness principle

$$
\|B(t) x\| \leq M(T)(\|x\|+\|A x\|) \quad \text { for } t \in[0, T] \text { and } x \in D(A),
$$

where $M(T)$ is some constant depending on $T>0$.

Our main result in this paper is stated as follows.

Theorem 3.2. Suppose that a closed linear operator $A$ in $X$ satisfies the Hille-Yosida condition (H.-Y.) and that a family $\{B(t): t \geq 0\}$ of 
linear operators in $X$ satisfies (H2). Then there exists a unique locally Lipschitz continuous integrated resolvent operator $\{R(t): t \geq 0\}$.

Proof. Let $\lambda_{0} \in \rho(A)$ with $\lambda_{0}>\max \{0, \omega\}$ and define an operator $K(t) \in B(X)$ by $K(t)=-B(t)\left(\lambda_{0}-A\right)^{-1}$ for $t \geq 0$. Then by (H2) the function $K(\cdot) x$ is of strong bounded variation on each finite interval $[0, T]$ for $x \in X$ and there exists a function $k \in L_{\text {loc }}^{1}\left(\mathbf{R}^{+} ; \mathbf{R}^{+}\right)$such that $\|K(t)\| \leq k(t)$ for almost every $t \geq 0$. Let $L(t)$ denote the resolvent kernel for $K(t)$, i.e.

$$
L(t) x=K(t) x-(L * K)(t) x
$$

for almost every $t \geq 0$ and $x \in X$. Then $L(\cdot) x$ is of strong bounded variation on each finite interval $[0, T]$ for $x \in X$ and $\|L(t)\| \leq r(t)$ for almost every $t \geq 0$, where $r \in L_{\text {loc }}^{1}\left(\mathbf{R}^{+} ; \mathbf{R}^{+}\right)$denotes the resolvent kernel of $k$, i.e., $r=k+r * k$.

Let $\{S(t): t \geq 0\}$ be a locally Lipschitz continuous integrated semigroup on $X$ generated by $A$. Since $L(\cdot) x \in B V_{\text {loc }}\left(\mathbf{R}^{+} ; X\right)$, Lemma 2.6 (ii) implies that a function $t \mapsto(d / d t) \int_{0}^{t} S(t-s) L(s) x d s$ is Lipschitz continuous on each finite interval $[0, T]$ for $x \in X$. Define an operator $V(t): X \rightarrow X$ for $t \geq 0$ by

$$
V(t) x=\frac{d}{d t} \int_{0}^{t} S(t-s) L(s) x d s \quad \text { for } x \in X .
$$

In view of (3.2) and $\|L(t)\| \leq r(t)$ for almost every $t \geq 0$, we have $V(t) \in B(X)$ for $t \geq 0$. Hence by the uniform boundedness principle, $\{V(t): t \geq 0\}$ is locally Lipschitz continuous. Since $V(0)=0$ by (3.1), from Lemma 2.6 (i) it follows that a function $t \mapsto \int_{0}^{t} V(t-s) g(s) d s$ is in $C^{1}$ for $g \in L^{1}(0, T ; X)$.

Let $x \in X$. Let $T>0$ be fixed arbitrarily and define an operator $\Phi: C([0, T] ; X) \rightarrow C([0, T] ; X)$ by

$$
(\Phi u)(t)=S(t) x-W(t) x+\frac{d}{d t}(V * u)(t)-\lambda_{0}(V * u)(t)
$$

for $u \in C([0, T] ; X)$ and $t \in[0, T]$, where $W(t) z=(S * L(\cdot) z)(t)$ for $t \in[0, T]$ and $z \in X$. Since $\{V(t): t \geq 0\}$ is locally Lipschitz continuous 
and $V(0)=0$, we have $\|V(t)\| \leq C_{T} T$ for $t \in[0, T]$ where $C_{T}$ is the Lipschitz constant of $\{V(t): t \in[0, T]\}$. Noting this and the inequality (2.9), one can verify by induction

$$
\begin{aligned}
& \left\|\left(\Phi^{n} u_{1}\right)(t)-\left(\Phi^{n} u_{2}\right)(t)\right\| \\
& \quad \leq\left(1+\lambda_{0} T\right)^{n} C_{T}^{n} \int_{0}^{t} \frac{(t-s)^{n-1}}{(n-1) !}\left\|u_{1}(s)-u_{2}(s)\right\| d s
\end{aligned}
$$

for $n=1,2, \ldots$, and $t \in[0, T]$. The fixed point theorem asserts that $\Phi$ has a unique fixed point $u_{x, T} \in C([0, T] ; X)$, namely,

$$
u_{x, T}(t)=S(t) x-W(t) x+\frac{d}{d t}\left(V * u_{x, T}\right)(t)-\lambda_{0}\left(V * u_{x, T}\right)(t)
$$

for $t \in[0, T]$. Define a function $u_{x} \in C([0, \infty) ; X)$ by $u_{x}(t)=u_{x, T}(t)$ if $0 \leq t \leq T$. Then $u_{x}$ is a unique element in $C([0, \infty) ; X)$ satisfying

$$
u_{x}(t)=S(t) x-W(t) x+\frac{d}{d t}\left(V * u_{x}\right)(t)-\lambda_{0}\left(V * u_{x}\right)(t)
$$

for $t \geq 0$.

For $t \geq 0$ we define an operator $R(t): X \rightarrow X$ by $R(t) x=u_{x}(t)$ for $x \in X$. Clearly, each $R(t)$ is a linear operator on $X$. To show $R(t) \in B(X)$, we consider a linear operator $T: X \rightarrow C([0, T] ; X)$ defined by $T x=R(\cdot) x$ for $x \in X$. Noting the inequality (2.9), by virtue of (3.5) we see that $T$ is closed. Thus by the closed graph theorem we conclude that $R(t) \in B(X)$.

Let us show that $\{R(t): t \geq 0\}$ is a locally Lipschitz continuous integrated resolvent operator. Obviously (r1) is satisfied.

Next we shall show (r2) and (r3). From [1, Proposition 3.3] and Lemma 3.1 it follows that

$$
\begin{gathered}
\int_{0}^{t} S(s) x d s \in D(A), \quad A \int_{0}^{t} S(s) x d s=S(t) x-t x \\
W(t) x \in D(A) \quad \text { and } \quad A W(t) x=V(t) x-\int_{0}^{t} L(s) x d s
\end{gathered}
$$


for $t \geq 0$ and $x \in X$. Integrating (3.7) and using the closedness of $A$ we have

$$
\begin{gathered}
\int_{0}^{t} W(s) x d s \in D(A) \quad \text { and } \\
A \int_{0}^{t} W(s) x d s=W(t) x-\int_{0}^{t} \int_{0}^{s} L(r) x d r d s
\end{gathered}
$$

for $t \geq 0$ and $x \in X$. Convolving both sides of (3.7) with $R(\cdot) x$ from the right side and noting Lemma 2.6 (i), we see that

$$
(A W * R(\cdot) x)(t)=(V * R(\cdot) x)(t)-\int_{0}^{t}(L * R(\cdot) x)(s) d s
$$

belongs to $C^{1}$. Since the equality $(V * R(\cdot) x)(t)=(d / d t)(W * R(\cdot) x)(t)$ holds, from the closedness of $A$ we deduce $(V * R(\cdot) x)(t) \in D(A)$ and

$$
\begin{aligned}
A(V * R(\cdot) x)(t) & =\frac{d}{d t}(A W * R(\cdot) x)(t) \\
& =\frac{d}{d t}(V * R(\cdot) x)(t)-(L * R(\cdot) x)(t)
\end{aligned}
$$

for $t \geq 0$ and $x \in X$. The combination of (3.5)-(3.9) with the closedness of $A$ shows (r2) and

$$
\begin{aligned}
A \int_{0}^{t} R(s) x d s= & S(t) x-t x-W(t) x+\int_{0}^{t} \int_{0}^{s} L(r) x d r d s \\
& +\frac{d}{d t}(V * R(\cdot) x)(t) \\
& -(L * R(\cdot) x)(t)-\lambda_{0}(V * R(\cdot) x)(t) \\
& +\lambda_{0} \int_{0}^{t}(L * R(\cdot) x)(s) d s \\
= & R(t) x-t x-\left(L *\left(R(\cdot) x-j_{1}(\cdot) x\right)\right)(t)+\lambda_{0}(L * U(\cdot) x)(t)
\end{aligned}
$$

where $U(t) x=\int_{0}^{t} R(s) x d s$ for $t \geq 0$ and $x \in X$.

Set

$$
w(t)=R(t) x-t x-A U(t) x-(B * U(\cdot) x)(t)
$$


for $t \geq 0$ and $x \in X$. Then $w(\cdot) \in C([0, \infty) ; X)$. Noting $B(t) y=$ $K(t) A y-\lambda_{0} K(t) y$ for $y \in D(A)$ and

$$
\begin{aligned}
B * U & =(K-L * K) * A U+L * K A * U-\lambda_{0} K * U \\
& =L * A U+L *\left(B+\lambda_{0} K\right) * U-\lambda_{0} K * U \\
& =L *(A U+B * U)+\lambda_{0}(L * K-K) * U \\
& =L *(A U+B * U)-\lambda_{0} L * U
\end{aligned}
$$

by (3.3), we have with (3.10)

$$
\begin{aligned}
w(t)= & \left(L *\left(R(\cdot) x-j_{1}(\cdot) x\right)\right)(t) \\
& -\lambda_{0}(L * U(\cdot) x)(t)-(B * U(\cdot) x)(t) \\
= & \left(L *\left(R(\cdot) x-j_{1}(\cdot) x-A U(\cdot) x-(B * U(\cdot) x)(\cdot)\right)\right)(t) \\
= & (L * w)(t),
\end{aligned}
$$

which implies

$$
\|w(t)\| \leq \int_{0}^{t} r(t-s)\|w(s)\| d s \quad \text { for } t \geq 0 .
$$

Therefore by Gronwall's inequality we have $w(t)=0$ for $t \geq 0$. This shows that $(\mathrm{r} 3)$ is satisfied.

To prove (r4), let $x \in D(A)$ and put

$$
y(t)=t x+\int_{0}^{t} R(s) A x d s+\int_{0}^{t} \int_{0}^{s} R(s-r) B(r) x d r d s
$$

for $t \geq 0$. Noting $y(t)-t x=\int_{0}^{t} R(t-s)\left(A x+\int_{0}^{s} B(r) x d r\right) d s$ for $t \geq 0$, by (2.6) (note that Lemma 2.5 holds if (r1), (r2) and (r3) are satisfied), we obtain $\int_{0}(y(s)-s x) d s \in C([0, \infty) ; Y)$ and

$$
\begin{aligned}
y(t)-t x= & A \int_{0}^{t}(y(s)-s x) d s \\
& +\int_{0}^{t} B(t-s) \int_{0}^{s}(y(r)-r x) d r d s \\
& +\int_{0}^{t} \int_{0}^{s}\left(A x+\int_{0}^{r} B(\xi) x d \xi\right) d r d s \\
= & A \int_{0}^{t} y(s) d s+\int_{0}^{t} B(t-s) \int_{0}^{s} y(r) d r d s
\end{aligned}
$$


for $t \geq 0$. Then putting $z(t)=y(t)-R(t) x$ for $t \geq 0$, we have

$$
z(t)=A \int_{0}^{t} z(s) d s+\int_{0}^{t} B(t-s) \int_{0}^{s} z(r) d r d s
$$

for $t \geq 0$. Replacing $U$ in (3.11) by $\int_{0}^{\cdot} z(s) d s$, we get

$$
\begin{aligned}
z(t)= & A \int_{0}^{t} z(s) d s+\left(L *\left(A \int_{0}^{\cdot} z(s) d s+B * \int_{0}^{\cdot} z(s) d s\right)\right)(t) \\
& -\lambda_{0}\left(L * \int_{0}^{\cdot} z(s) d s\right)(t) \\
= & A \int_{0}^{t} z(s) d s+(L * z)(t)-\lambda_{0}\left(L * \int_{0}^{r} z(s) d s\right)(t)
\end{aligned}
$$

for $t \geq 0$. Integrating this twice with the closedness of $A$, we obtain

$$
\begin{aligned}
v(t)= & A \int_{0}^{t} v(s) d s+\int_{0}^{t} \int_{0}^{s}(L * z)(r) d r d s \\
& -\lambda_{0} \int_{0}^{t} \int_{0}^{s}\left(L * \int_{0}^{\cdot} z(\eta) d \eta\right)(r) d r d s
\end{aligned}
$$

where $v(t)=\int_{0}^{t} \int_{0}^{s} z(r) d r d s$ for $t \geq 0$. Since $A$ is the generator of an integrated semigroup $\{S(t): t \geq 0\}$ on $X,[\mathbf{1 3}$, Theorem 4.1] implies

$$
\begin{aligned}
v(t) & =\int_{0}^{t} S(t-s)\left(L *\left(z(\cdot)-\lambda_{0} \int_{0} z(\eta) d \eta\right)\right)(s) d s \\
& =\left(W *\left(z(\cdot)-\lambda_{0} \int_{0} z(\eta) d \eta\right)\right)(t)
\end{aligned}
$$

for $t \geq 0$. Differentiating this twice, we have $z(t)=(d / d t)(V *(z(\cdot)-$ $\left.\left.\lambda_{0} \int_{0} z(\eta) d \eta\right)\right)(t)$ for $t \geq 0$. Let $T>0$ be any positive number. We note the inequality (2.9) and estimate the above equation to get

$$
\begin{aligned}
\|z(t)\| & \leq C_{T} \int_{0}^{t}\left(\|z(s)\|+\lambda_{0} \int_{0}^{s}\|z(r)\| d r\right) d s \\
& \leq C_{T}\left(1+\lambda_{0} T\right) \int_{0}^{t}\|z(s)\| d s
\end{aligned}
$$


for $t \in[0, T]$. Thus Gronwall's inequality gives $z(t)=0$ on $[0, T]$. Since $T>0$ is arbitrary, $z(t)=0$ for $t \geq 0$. This proves $(\mathrm{r} 4)$.

Finally we shall show the local Lipschitz continuity of $\{R(t): t \geq 0\}$. Since $\{S(t): t \geq 0\},\{W(t): t \geq 0\}$ and $\{V(t): t \geq 0\}$ are locally Lipschitz continuous, in view of (3.5) and (2.10) we have

$$
\begin{aligned}
\| R(t+ & h) x-R(t) x \| \\
\leq & h\left(M_{T}+\sup _{s \in[0, T]}\|R(s)\| \cdot C_{T}+2 \lambda_{0} C_{T} T \cdot \sup _{s \in[0, T]}\|R(s)\|\right)\|x\| \\
& +C_{T} \int_{0}^{t}\|R(r+h) x-R(r) x\| d r
\end{aligned}
$$

for $t, h, t+h \in[0, T]$ and $x \in X$ where $M_{T}$ is the maximum of the Lipschitz constants of $\{S(t): t \in[0, T]\}$ and $\{W(t): t \in[0, T]\}$. Then Gronwall's inequality implies the desired conclusion.

Theorems 2.7 and 3.2 are applied to two Cauchy problems:

$$
\begin{gathered}
u^{\prime \prime}(t)-A u^{\prime}(t)-B u(t)=f(t) \\
\text { for } t \in[0, T], u(0)=x \text { and } u^{\prime}(0)=y ; \\
u^{\prime}(t)=A\left(u(t)+\int_{0}^{t} F(t-s) u(s) d s\right) \\
+K u(t)+f(t) \quad \text { for } t \in[0, T] \text { and } u(0)=x .
\end{gathered}
$$

The complete second order equation (SE) is an abstract form of the linear strongly damped wave equation and the Klein-Gordon equation, etc. (see [14]). Also, a partial integrodifferential equation from heat conduction in materials with memory

$$
\begin{aligned}
u_{t}(t, x)= & \left(u(t, x)+\int_{0}^{t} b(t-s) u(s, x) d s\right)_{x x} \\
& +f(t, x), \quad t \in[0, T], x \in[0,1], \\
u(0, x)= & u_{0}(x), \quad x \in[0,1], \\
u(t, 0)= & u(t, 1)=0, \quad t \in[0, T]
\end{aligned}
$$


can be formulated as the abstract integrodifferential equation (IE).

Definition 3.1. We say that a function $u:[0, T] \rightarrow X$ is a classical solution to (SE) if $u \in C^{2}([0, T] ; X), u(t) \in D(B), u^{\prime}(t) \in D(A), B u(t)$ and $A u^{\prime}(t)$ are continuous and (SE) is satisfied for $t \in[0, T]$.

Theorem 3.3. Let $A$ be a closed linear operator in $X$ satisfying the Hille-Yosida condition (H.-Y.). Suppose that $B$ is a linear operator in $X$ with $D(A) \subset D(B)$ and a bounded linear operator from $Y$ into $X$, where $Y$ is a Banach space $D(A)$ equipped with the graph norm of $A$. If $x \in D(B), y \in D(A), f \in W^{1,1}(0, T ; X)$ and $B x+A y+f(0) \in \overline{D(A)}$, the complete second order equation (SE) has a unique classical solution $v$ which satisfies

$$
\begin{aligned}
& \|v(t)\| \leq C\left(\|x\|+\|y\|+\int_{0}^{t}\|B x+f(s)\| d s\right) \\
& \left\|v^{\prime}(t)\right\| \leq C\left(\|y\|+\int_{0}^{t}\|B x+f(s)\| d s\right) \\
& \left\|v^{\prime \prime}(t)\right\| \leq C\left(\|B x+A y+f(0)\|+\int_{0}^{t}\left\|B y+f^{\prime}(s)\right\| d s\right)
\end{aligned}
$$

for $t \in[0, T]$, where $C$ is a constant independent of $x, y$ and $f$.

Proof. Theorem 3.2 shows that the following integrodifferential equation

$$
\begin{aligned}
& u^{\prime}(t)=A u(t)+\int_{0}^{t} B u(s) d s+B x+f(t) \quad \text { for } t \in[0, T] \\
& u(0)=y
\end{aligned}
$$

admits a locally Lipschitz continuous integrated resolvent operator $\{R(t): t \geq 0\}$. Therefore, by Theorem 2.7 (ii) and the assumption we see that (ID) has a unique classical solution $u$ which is given by the variation of constants formula

$$
u(t)=\frac{d}{d t}\left(R(t) y+\int_{0}^{t} R(t-s)(B x+f(s)) d s\right)
$$


for $t \in[0, T]$. Then a function $v$ defined by $v(t)=x+\int_{0}^{t} u(s) d s$ for $t \in[0, T]$ is a classical solution to (SE). Conversely if $v$ is a classical solution to (SE), a function $u:=v^{\prime}$ is a classical solution to (ID). The estimates (3.13)-(3.15) of a classical solution can be obtained by differentiating $v$ and using the property $(\mathrm{r} 4)$ and the local Lipschitz continuity of $\{R(t): t \geq 0\}$.

Remark 3.1. Theorem 3.3 extends [15, Theorem 3.3] to the case of nondensely defined operators $A$ and $B$. It should be noted that we do not assume the closedness of $B$ in $X$.

Next we consider the following integrodifferential equation

$$
\begin{aligned}
u^{\prime}(t)= & A\left(u(t)+\int_{0}^{t} F(t-s) u(s) d s\right) \\
& +K u(t)+f(t) \quad \text { for } t \in[0, T] \\
u(0)= & x,
\end{aligned}
$$

where $K$ and $F(t)$ for $t \geq 0$ are bounded linear operators on $X$.

Definition 3.2. A function $u:[0, T] \rightarrow X$ is a classical solution to $(\mathrm{IE})$ if $u(t)+\int_{0}^{t} F(t-s) u(s) \in D(A)$ for $t \in[0, T], u \in C^{1}([0, T] ; X)$ and $u$ satisfies (IE).

Theorem 3.4. Let $A$ be a closed linear operator in $X$ satisfying the Hille-Yosida condition (H.-Y.). Suppose $K, F(t) \in B(X)$ for $t \geq 0$ and $F(\cdot) z \in W_{\text {loc }}^{1,1}\left(\mathbf{R}^{+} ; X\right)$ for $z \in X$. If $x \in D(A), f \in W^{1,1}(0, T ; X)$ and $(A+K) x+F(0) x+f(0) \in \overline{D(A)}$, the integrodifferential equation (IE) has a unique classical solution $u$ satisfying

$$
\|u(t)\| \leq C\left(\|x\|+\int_{0}^{t}\|f(s)\| d s\right)
$$

for $t \in[0, T]$ where $C$ is a constant independent of $x$ and $f$.

Proof. Define two operators in $X \times X$ with domain $X \times D(A)$ by

$$
\mathcal{A}_{0}=\left(\begin{array}{ll}
O & A \\
O & A
\end{array}\right) \quad \text { and } \quad \mathcal{B}(t)=\left(\begin{array}{cc}
O & O \\
F(t) K & F(t) A
\end{array}\right)
$$


for $t \geq 0$ respectively. It is shown in [8, pp. 88-89] that $\mathcal{A}_{0}$ generates a locally Lipschitz continuous integrated semigroup on $X \times X$. Since $K \in B(X)$, a bounded perturbation theorem for locally Lipschitz continuous integrated semigroups ([12, Proposition 3.3]) shows that an operator $\mathcal{A}$ defined by

$$
\mathcal{A}=\left(\begin{array}{ll}
K & A \\
K & A
\end{array}\right)
$$

generates a locally Lipschitz continuous integrated semigroup on $X \times X$, i.e. a closed linear operator $\mathcal{A}$ in $X \times X$ satisfies the Hille-Yosida condition (H.-Y.). Moreover $\mathcal{B}(t)$ is a bounded linear operator from $[D(\mathcal{A})]$ into $X \times X$ for $t \geq 0$ and $\mathcal{B}(\cdot)\left(\begin{array}{l}u \\ v\end{array}\right) \in W_{\text {loc }}^{1,1}\left(\mathbf{R}^{+} ; X \times X\right)$ for $(u, v) \in D(\mathcal{A})=X \times D(A)$, where $[D(\mathcal{A})]$ is a Banach space $D(\mathcal{A})$ equipped with the graph norm of $\mathcal{A}$. Therefore Theorem 3.2 shows that the integrodifferential equation

$$
\begin{aligned}
\mathcal{U}^{\prime}(t) & =\mathcal{A} \mathcal{U}(t)+\int_{0}^{t} \mathcal{B}(t-s) \mathcal{U}(s) d s+\mathcal{F}(t) \quad \text { for } t \in[0, T] \\
\mathcal{U}(0) & =\mathcal{X}
\end{aligned}
$$

admits a locally Lipschitz continuous integrated resolvent operator $\{\mathbf{R}(t): t \geq 0\}$ on $X \times X$. Here we put $\mathcal{F}(t)=(f(t), f(t)+F(t) x+$ $\left.\int_{0}^{t} F(t-s) f(s) d s\right)$ and $\mathcal{X}=(x, x)$. Then from Theorem 2.7 (ii) and assumptions it follows that $(\mathcal{I} E)$ has a unique classical solution $\mathcal{U}$. It is easy to see that the first component of $\mathcal{U}$ is a classical solution to (IE). Conversely if $u$ is a classical solution to (IE), a function $\mathcal{U}(t):=\left(u(t), u(t)+\int_{0}^{t} F(t-s) u(s) d s\right)$ for $t \in[0, T]$ is a classical solution to $(\mathcal{I} E)$. The estimate (3.16) of a classical solution to (IE) follows from the variation of constants formula which is given in terms of $\{\mathbf{R}(t): t \geq 0\}$.

Remark 3.2. Theorem 3.4 gives a slight improvement on $[8$, Theorem $3.2]$.

4. Examples. As illustrations of our abstract theory, we will give two examples of hyperbolic partial integrodifferential equations.

We prepare the following theorem. 
Theorem 4.1. Suppose that a closed linear operator $A$ in $X$ satisfies the Hille-Yosida condition (H.-Y.). Let $F(\cdot):[0, T] \rightarrow B(X)$ be a function such that $F(\cdot) v \in C^{1}([0, T] ; X)$ for each $v \in X$. Let $\{K(t): t \geq 0\}$ be subject to $(\mathrm{H} 2)$ and let $f \in W^{1,1}(0, T ; X)$. If $x \in D(A)$ and $(A+F(0)) x+f(0) \in \overline{D(A)}$, then the Cauchy problem

$$
\begin{aligned}
& u^{\prime}(t)=(A+F(t)) u(t)+\int_{0}^{t} K(t-s) u(s) d s+f(t) \quad \text { for } t \in[0, T] \\
& u(0)=x
\end{aligned}
$$

has a unique classical solution $u \in C^{1}([0, T] ; X) \cap C([0, T] ; Y)$.

Proof. Consider a Banach space $\mathcal{X}=\left\{u \in C^{1}([0, T] ; X): u(0)=x\right\}$ with norm $|\cdot|$ defined by $|u|=\sup _{t \in[0, T]}\left\{\|u(t)\|+\left\|u^{\prime}(t)\right\|\right\}$ for $u \in \mathcal{X}$. Define an operator $J: \mathcal{X} \rightarrow \mathcal{X}$ by

$$
(J u)(t)=R^{\prime}(t) x+\frac{d}{d t} \int_{0}^{t} R(t-s)(F(s) u(s)+f(s)) d s
$$

for $t \in[0, T]$ and $u \in \mathcal{X}$, where $\{R(t): t \geq 0\}$ is a locally Lipschitz continuous integrated resolvent operator which the following integrodifferential equation admits by Theorem 3.2

$$
\begin{aligned}
u^{\prime}(t) & =A u(t)+\int_{0}^{t} K(t-s) u(s) d s+f(t) \quad \text { for } t \in[0, T] \\
u(0) & =x .
\end{aligned}
$$

By $(\mathrm{r} 4)$ the right side of $(4.2)$ is equal to

$$
\begin{aligned}
x+ & R(t)((A+F(0)) x+f(0)) \\
& +\int_{0}^{t} R(t-s)\left(K(s) x+F^{\prime}(s) u(s)+F(s) u^{\prime}(s)+f^{\prime}(s)\right) d s,
\end{aligned}
$$

which is a $C^{1}$-function of $t$ because of $(A+F(0)) x+f(0) \in \overline{D(A)}$ and Lemma 2.6 (i). Moreover (4.3) at $t=0$ is equal to $x$. Therefore $J$ is well-defined. Here denote the derivative of $F(t) v$ by $F^{\prime}(t) v$ for $t \in[0, T]$ and $v \in X$. Differentiating (4.3) yields

$$
\begin{aligned}
\frac{d}{d t}(J u)(t)= & R^{\prime}(t)((A+F(0)) x+f(0)) \\
+\frac{d}{d t} \int_{0}^{t} R(t-s)( & K(s) x+F^{\prime}(s) u(s) \\
& \left.+F(s) u^{\prime}(s)+f^{\prime}(s)\right) d s
\end{aligned}
$$


for $t \in[0, T]$. Noting the inequality (2.9), from (4.3) and (4.4) we find

$$
\begin{aligned}
& \left\|\left(J^{n} u_{1}\right)(t)-\left(J^{n} u_{2}\right)(t)\right\|+\left\|\left(J^{n} u_{1}\right)^{\prime}(t)-\left(J^{n} u_{2}\right)^{\prime}(t)\right\| \\
& \quad \leq K_{T}^{n} \int_{0}^{t} \frac{(t-s)^{n-1}}{(n-1) !}\left(\left\|u_{1}(s)-u_{2}(s)\right\|+\left\|u_{1}^{\prime}(s)-u_{2}^{\prime}(s)\right\|\right) d s
\end{aligned}
$$

for $t \in[0, T]$ and $n=1,2, \ldots$, where $K_{T}=\left(C_{T}+\sup _{s \in[0, T]}\|R(s)\|\right)$ $\cdot \max \left(\sup _{s \in[0, T]}\|F(s)\|, \sup _{s \in[0, T]}\left\|F^{\prime}(s)\right\|\right)$ and $C_{T}$ is a Lipschitz constant of $\{R(t): t \in[0, T]\}$. Hence the fixed point theorem asserts that $J$ has a unique fixed point $u \in C^{1}([0, T] ; X)$ with $u(0)=x$ and so Theorem 2.4 (ii) shows that $u$ is a classical solution to the problem (4.1). The uniqueness of classical solutions follows from that of fixed points of $J$ and Proposition 2.3 (ii).

Example 4.1. We consider a linear first-order hyperbolic integrodifferential equation in one space variable, namely,

$$
\begin{aligned}
u_{t}(t, x)+a(x) & u_{x}(t, x)+b(t, x) u(t, x) \\
= & \int_{0}^{t}\left\{p(t-s, x) u_{x}(s, x)+q(t-s, x) u(s, x)\right\} d s \\
& +f(t, x), \quad t \in[0, T], x \in[0,1], \\
u(0, x)= & u_{0}(x), \quad x \in[0,1], \\
u(t, 0)= & u(t, 1), \quad t \in[0, T] .
\end{aligned}
$$

Here $a$ is a positive continuous function on $[0,1], b \in C^{1}([0, T] ; C[0,1])$, $p, q \in B V_{\mathrm{loc}}([0, \infty) ; C[0,1])$ and $f \in W^{1,1}(0, T ; C[0,1])$.

Let $X=C[0,1]$ with norm $\|u\|=\sup _{x \in[0,1]}|u(x)|$ for $u \in X$ and define an operator $A$ and two families $\{C(t): t \in[0, T]\}$ and $\{B(t): t \geq 0\}$ of operators in $X$ by

$$
\begin{aligned}
& D(A)=\left\{u \in C^{1}[0,1]: u(0)=u(1)\right\} \\
& (A u)(x)=-a(x) u^{\prime}(x) \quad \text { for } x \in[0,1] \text { and } u \in D(A), \\
& D(C(t))=C[0,1] \\
& (C(t) u)(x)=-b(t, x) u(x) \quad \text { for } x \in[0,1], u \in C[0,1] \text { and } t \in[0, T] \\
& \text { and }
\end{aligned}
$$


$D(B(t))=C^{1}[0,1]$

$(B(t) u)(x)=p(t, x) u^{\prime}(x)+q(t, x) u(x)$

for $x \in[0,1], u \in C^{1}[0,1]$ and $t \geq 0$,

respectively. Then the integrodifferential equation (4.5) can be written in the abstract form

$$
\begin{aligned}
u^{\prime}(t) & =(A+C(t)) u(t)+\int_{0}^{t} B(t-s) u(s) d s+f(t) \quad \text { for } t \in[0, T] \\
u(0) & =u_{0} .
\end{aligned}
$$

For $g \in X$ and $\lambda>0$, the unique solution $u$ of $\lambda u-A u=g$ is given by

$$
u(x)=c e^{-\lambda d(x)}+\int_{0}^{x} e^{-\lambda(d(x)-d(y))} \frac{g(y)}{a(y)} d y
$$

where $d(x)=\int_{0}^{x}(1 / a(y)) d y$ and $c=\left(e^{\lambda d(1)}-1\right)^{-1} \int_{0}^{1} e^{\lambda d(y)} g(y) / a(y) d y$. Since $(1 / \lambda)(d / d y) e^{\lambda d(y)}=e^{\lambda d(y)} / a(y)>0$, we have

$$
\begin{aligned}
|u(x)| \leq & e^{-\lambda d(x)}\left(e^{\lambda d(1)}-1\right)^{-1} \int_{0}^{1} \frac{1}{\lambda}\left(\frac{d}{d y} e^{\lambda d(y)}\right)|g(y)| d y \\
& +e^{-\lambda d(x)} \int_{0}^{x} \frac{1}{\lambda}\left(\frac{d}{d y} e^{\lambda d(y)}\right)|g(y)| d y \leq \frac{\|g\|}{\lambda}
\end{aligned}
$$

and hence $(0, \infty) \subset \rho(A)$ and $\left\|(\lambda-A)^{-1}\right\| \leq 1 / \lambda$ for $\lambda>0$. Moreover it is clear that $\{B(t): t \geq 0\}$ is a family of bounded linear operators from $[D(A)]$ into $X$ satisfying $B(\cdot) x \in B V_{\text {loc }}\left(\mathbf{R}^{+} ; X\right)$ for $x \in D(A)$, where $[D(A)]$ is a Banach space $D(A)$ equipped with the graph norm of $A$. Since $\overline{D(A)}=\{u \in C[0,1]: u(0)=u(1)\}$, by Theorem 4.1 the problem (4.5) has a unique classical solution $u \in C^{1}([0, T] ; C[0,1])$ if $u_{0} \in C^{1}[0,1], u_{0}(0)=u_{0}(1)$ and $-a(0) u_{0}^{\prime}(0)-b(0,0) u_{0}(0)+f(0,0)=$ $-a(1) u_{0}^{\prime}(1)-b(0,1) u_{0}(1)+f(0,1)$. 
Example 4.2. We deal with the initial-boundary value problem

$$
\begin{aligned}
u_{t t}(t, x)= & u_{x x}(t, x)+b(t, x) u_{x}(t, x)+c(t, x) u(t, x) \\
& +\int_{0}^{t}\left\{p(t-s, x) u_{x x}(s, x)+q(t-s, x) u_{x}(s, x)\right. \\
& \quad+r(t-s, x) u(s, x)\} d s \\
& +f(t, x), \quad t \in[0, T], x \in[0,1], \\
u(t, 0)= & u(t, 1)=0, \quad t \in[0, T], \\
u(0, x)= & u_{0}(x), \quad u_{t}(0, x)=u_{1}(x), \quad x \in[0,1],
\end{aligned}
$$

where $b, c \in C^{1}([0, T] ; C[0,1]), f \in W^{1,1}(0, T ; C[0,1])$ and $p, q, r \in$ $B V_{\text {loc }}([0, \infty) ; C[0,1])$.

Let $X=C[0,1]$ with norm $\|u\|=\sup _{x \in[0,1]}|u(x)|$ for $u \in X$. Let $V=\left\{u \in C^{1}[0,1]: u(0)=u(1)=0\right\}$ with norm $\|u\|_{V}=\|u\|+\left\|u^{\prime}\right\|$ for $u \in V$.

Consider an operator $A$ and two families $\{F(t): t \in[0, T]\}$ and $\{K(t): t \geq 0\}$ in $X$ defined by

$$
\begin{aligned}
& D(A)=\left\{u \in C^{2}[0,1]: u(0)=u(1)=0\right\} \\
& (A u)(x)=u^{\prime \prime}(x) \quad \text { for } x \in[0,1] \text { and } u \in D(A), \\
& D(F(t))=V \\
& (F(t) u)(x)=b(t, x) u^{\prime}(x)+c(t, x) u(x) \\
& \quad \text { for } x \in[0,1], u \in V \text { and } t \in[0, T]
\end{aligned}
$$

and

$$
\begin{aligned}
& D(K(t))=D(A) \\
& (K(t) u)(x)=p(t, x) u^{\prime \prime}(x)+q(t, x) u^{\prime}(x)+r(t, x) u(x) \\
& \quad \text { for } x \in[0,1], u \in D(A) \text { and } t \geq 0
\end{aligned}
$$

respectively. 
Then the integrodifferential equation (4.6) can be written in the abstract form:

(SVE)

$$
\begin{aligned}
& u^{\prime \prime}(t)=(A+F(t)) u(t)+\int_{0}^{t} K(t-s) u(s) d s+f(t) \quad \text { for } t \in[0, T] \\
& u(0)=u_{0} \quad \text { and } \quad u^{\prime}(0)=u_{1} .
\end{aligned}
$$

We will try to convert (SVE) in $X$ into the first-order system in the Banach space $\left(V \times X,\|\cdot\|_{V}+\|\cdot\|\right)$

$$
\begin{aligned}
\left(\frac{d}{d t}\right)\left(\begin{array}{l}
u(t) \\
v(t)
\end{array}\right)= & (\mathcal{A}+\mathcal{F}(t))\left(\begin{array}{l}
u(t) \\
v(t)
\end{array}\right)+\int_{0}^{t} \mathcal{K}(t-s)\left(\begin{array}{l}
u(s) \\
v(s)
\end{array}\right) d s \\
& +\mathcal{G}(t) \quad \text { for } t \in[0, T] \\
\left(\begin{array}{c}
u(0) \\
v(0)
\end{array}\right)= & \left(\begin{array}{l}
u_{0} \\
u_{1}
\end{array}\right),
\end{aligned}
$$

where

$$
\begin{gathered}
\mathcal{A}=\left(\begin{array}{cc}
O & I \\
A & O
\end{array}\right), \quad \mathcal{F}(t)=\left(\begin{array}{cc}
O & O \\
F(t) & O
\end{array}\right), \\
\mathcal{K}(t)=\left(\begin{array}{cc}
O & O \\
K(t) & O
\end{array}\right) \quad \text { and } \quad \mathcal{G}(t)=\left(\begin{array}{c}
0 \\
f(t)
\end{array}\right) .
\end{gathered}
$$

Since $F(t) \in B(V, X)$ (the set of all bounded linear operators from $V$ into $X)$, the operator $\mathcal{F}(t)$ with domain $V \times X$ belongs to $B(V \times$ $X, V \times X)$ for $t \in[0, T]$ and, for $(v, w) \in V \times X, \mathcal{F}(t)\left(\begin{array}{c}v \\ w\end{array}\right)=\left(\begin{array}{c}0 \\ F(t) v\end{array}\right)$ is continuously differentiable in $t \in[0, T]$ in the topology of $V \times X$. Also the operator $\mathcal{K}(t)$ with domain $D(A) \times X$ is a bounded linear operator from $[D(\mathcal{A})]$ into $V \times X$, where $[D(\mathcal{A})]$ is a Banach space $D(\mathcal{A})$ equipped with the graph norm of $\mathcal{A}$, because for $(u, v) \in D(\mathcal{A})=D(A) \times V$ we have

$$
\begin{aligned}
\left\|\mathcal{K}(t)\left(\begin{array}{c}
u \\
v
\end{array}\right)\right\|_{V \times X} & =\|K(t) u\| \\
& \leq c_{T}\left(\|u\|_{V}+\|A u\|\right) \\
& \leq c_{T}\left(\left\|\left(\begin{array}{l}
u \\
v
\end{array}\right)\right\|_{V \times X}+\left\|\mathcal{A}\left(\begin{array}{l}
u \\
v
\end{array}\right)\right\|_{V \times X}\right) .
\end{aligned}
$$


Here we put $c_{T}=\sup \{|p(t, x)|+|q(t, x)|+|r(t, x)|: t \in[0, T], x \in[0,1]\}$. From the assumption it follows that $\mathcal{K}(t)\left(\begin{array}{l}u \\ v\end{array}\right)=\left(\begin{array}{c}0 \\ K(t) u\end{array}\right) \in B V_{\text {loc }}\left(\mathbf{R}^{+} ; V \times\right.$ $X)$ for $(u, v) \in D(\mathcal{A})=D(A) \times V$. Therefore if the operator $\mathcal{A}$ in $V \times X$ satisfies the Hille-Yosida condition (H.-Y.), we can apply Theorem 4.1 to the problem (4.7). Noting $\overline{D(A)}^{V}$ (the closure of $D(A)$ in $\left.V\right)=V$ and $\bar{V}=\{u \in C[0,1]: u(0)=u(1)=0\}$, we have

Theorem 4.2. Let $u_{0} \in C^{2}[0,1]$ and $u_{1} \in C^{1}[0,1]$. If

$$
\begin{aligned}
u_{0}(0) & =u_{0}(1)=u_{1}(0)=u_{1}(1) \\
& =u_{0}^{\prime \prime}(0)+b(0,0) u_{0}^{\prime}(0)+f(0,0) \\
& =u_{0}^{\prime \prime}(1)+b(0,1) u_{0}^{\prime}(1)+f(0,1)=0,
\end{aligned}
$$

the problem (4.6) has a unique classical solution $u \in C^{2}([0, T] ; C[0,1])$.

It remains to show

Proposition 4.3. The operator $\mathcal{A}=\left(\begin{array}{ll}O & I \\ A & O\end{array}\right)$ with domain $D(A) \times$ $V$ satisfies that there exists a positive number $\omega$ such that $(\omega, \infty) \subset$ $\rho(\mathcal{A})$ and

$$
\left\|(\lambda-\mathcal{A})^{-n}\left(\begin{array}{l}
f \\
g
\end{array}\right)\right\|_{V \times X} \leq\left(2+\omega^{-1}\right)(\lambda-\omega)^{-n}\left(\|f\|_{V}+\|g\|\right)
$$

for $\lambda>\omega,(f, g) \in V \times X$ and $n=0,1,2, \ldots$.

Proof. A simple computation shows

$$
\begin{array}{r}
\left(\lambda^{2}-A\right)^{-1} v(x)=\frac{e^{\lambda x}-e^{-\lambda x}}{2 \lambda\left(e^{\lambda}-e^{-\lambda}\right)} \int_{0}^{1}\left\{e^{\lambda(1-t)}-e^{-\lambda(1-t)}\right\} v(t) d t \\
-\frac{1}{2 \lambda} \int_{0}^{x}\left\{e^{\lambda(x-t)}-e^{-\lambda(x-t)}\right\} v(t) d t \\
=\frac{1}{2 \lambda}\left[\sum _ { k = 0 } ^ { \infty } \int _ { 0 } ^ { 1 } \left\{e^{-\lambda(2 k-x+t)}-e^{-\lambda(2 k+x+t)}\right.\right. \\
\left.-e^{-\lambda(2 k+2-x-t)}+e^{-\lambda(2 k+2+x-t)}\right\} v(t) d t \\
\left.-\int_{0}^{x}\left\{e^{-\lambda(t-x)}-e^{-\lambda(x-t)}\right\} v(t) d t\right]
\end{array}
$$


for $\lambda>0$ and $v \in X$.

Integration by parts gives

$$
\begin{array}{r}
\left(\lambda^{2}-A\right)^{-1} v(x)=\frac{e^{\lambda x}-e^{-\lambda x}}{2\left(e^{\lambda}-e^{-\lambda}\right)} \int_{0}^{1}\left\{e^{\lambda(1-t)}+e^{-\lambda(1-t)}\right\} w(t) d t \\
-\frac{1}{2} \int_{0}^{x}\left\{e^{\lambda(x-t)}+e^{-\lambda(x-t)}\right\} w(t) d t \\
=\frac{1}{2}\left[\sum _ { k = 0 } ^ { \infty } \int _ { 0 } ^ { 1 } \left\{e^{-\lambda(2 k-x+t)}-e^{-\lambda(2 k+x+t)}\right.\right. \\
\left.+e^{-\lambda(2 k+2-x-t)}-e^{-\lambda(2 k+2+x-t)}\right\} w(t) d t \\
\left.-\int_{0}^{x}\left\{e^{-\lambda(t-x)}+e^{-\lambda(x-t)}\right\} w(t) d t\right]
\end{array}
$$

where $w(t)=\int_{0}^{t} v(s) d s$ for $t \in[0,1], \lambda>0$ and $v \in X$.

Let $\lambda>0$ and $f \in V$. Differentiating (4.9) in $x$ and integrating by parts, we have

$$
\begin{aligned}
\left(\frac{d}{d x}\right)\left(\lambda^{2}-A\right)^{-1} f(x)= & \frac{e^{\lambda x}+e^{-\lambda x}}{2 \lambda\left(e^{\lambda}-e^{-\lambda}\right)} \int_{0}^{1}\left\{e^{\lambda(1-t)}+e^{-\lambda(1-t)}\right\} f^{\prime}(t) d t \\
& -\frac{1}{2 \lambda} \int_{0}^{x}\left\{e^{\lambda(x-t)}-e^{-\lambda(x-t)}\right\} f^{\prime}(t) d t
\end{aligned}
$$

Differentiating this again, we obtain

$$
\begin{array}{r}
A\left(\lambda^{2}-A\right)^{-1} f(x) \\
=\frac{1}{2}\left[\sum _ { k = 0 } ^ { \infty } \int _ { 0 } ^ { 1 } \left\{e^{-\lambda(2 k-x+t)}-e^{-\lambda(2 k+x+t)}\right.\right. \\
\left.+e^{-\lambda(2 k+2-x-t)}-e^{-\lambda(2 k+2+x-t)}\right\} f^{\prime}(t) d t \\
\left.\quad-\int_{0}^{x}\left\{e^{-\lambda(t-x)}+e^{-\lambda(x-t)}\right\} f^{\prime}(t) d t\right] .
\end{array}
$$


We differentiate (4.9) in $\lambda, n$-times, to get (4.12)

$$
\begin{aligned}
& \left(-\frac{d}{d \lambda}\right)^{n} \lambda\left(\lambda^{2}-A\right)^{-1} v(x) \\
& =\frac{1}{2}\left[\sum _ { k = 0 } ^ { \infty } \int _ { 0 } ^ { 1 } \left\{(2 k-x+t)^{n} e^{-\lambda(2 k-x+t)}\right.\right. \\
& -(2 k+x+t)^{n} e^{-\lambda(2 k+x+t)} \\
& -(2 k+2-x-t)^{n} e^{-\lambda(2 k+2-x-t)} \\
& \left.+(2 k+2+x-t)^{n} e^{-\lambda(2 k+2+x-t)}\right\} v(t) d t \\
& \left.-\int_{0}^{x}\left\{(t-x)^{n} e^{-\lambda(t-x)}-(x-t)^{n} e^{-\lambda(x-t)}\right\} v(t) d t\right] \\
& =\frac{1}{2}\left[\sum _ { k = 0 } ^ { \infty } \left\{\int_{2 k-x}^{2 k-x+1} s^{n} e^{-\lambda s} v(s+x-2 k) d s\right.\right. \\
& -\int_{2 k+x}^{2 k+x+1} s^{n} e^{-\lambda s} v(s-x-2 k) d s \\
& -\int_{2 k+1-x}^{2 k+2-x} s^{n} e^{-\lambda s} v(2 k+2-x-s) d s \\
& \left.+\int_{2 k+1+x}^{2 k+2+x} s^{n} e^{-\lambda s} v(2 k+2+x-s) d s\right\} \\
& \left.-\int_{-x}^{0} s^{n} e^{-\lambda s} v(s+x) d s+\int_{0}^{x} s^{n} e^{-\lambda s} v(x-s) d s\right] \\
& =\frac{1}{2}\left[\int_{0}^{1-x} s^{n} e^{-\lambda s} v(s+x) d s+\int_{0}^{x} s^{n} e^{-\lambda s} v(x-s) d s\right. \\
& +\sum_{k=1}^{\infty} \int_{2 k-x}^{2 k-x+1} s^{n} e^{-\lambda s} v(s+x-2 k) d s \\
& -\sum_{k=0}^{\infty}\left\{\int_{2 k+x}^{2 k+1+x} s^{n} e^{-\lambda s} v(s-x-2 k) d s\right. \\
& +\int_{2 k+1-x}^{2 k+2-x} s^{n} e^{-\lambda s} v(2 k+2-x-s) d s \\
& \left.\left.-\int_{2 k+1+x}^{2 k+2+x} s^{n} e^{-\lambda s} v(2 k+2+x-s) d s\right\}\right] \text {. }
\end{aligned}
$$


Estimating the integrals in the above equality we find

$$
\begin{aligned}
& \left|\left(-\frac{d}{d \lambda}\right)^{n} \lambda\left(\lambda^{2}-A\right)^{-1} v(x)\right| \\
& \leq \frac{1}{2}\left\{\int_{0}^{1-x}+\int_{0}^{x}+\sum_{k=1}^{\infty} \int_{2 k-x}^{2 k+1-x}\right. \\
& \left.\quad+\sum_{k=0}^{\infty}\left(\int_{2 k+x}^{2 k+1+x}+\int_{2 k+1-x}^{2 k+2-x}+\int_{2 k+1+x}^{2 k+2+x}\right)\right\} s^{n} e^{-\lambda s} d s \cdot\|v\| \\
& \quad=\int_{0}^{\infty} s^{n} e^{-\lambda s} d s \cdot\|v\|=\frac{n !}{\lambda^{n+1}}\|v\|
\end{aligned}
$$

for $\lambda>0, v \in X$ and $n=0,1,2, \ldots$.

Noting $f(0)=f(1)=0$ for $f \in V$ and differentiating (4.12) in $x$, we have

$$
\left|\left(\frac{d}{d x}\right)\left(-\frac{d}{d \lambda}\right)^{n} \lambda\left(\lambda^{2}-A\right)^{-1} f(x)\right| \leq \frac{n !}{\lambda^{n+1}}\left\|f^{\prime}\right\|
$$

for $\lambda>0, f \in V$ and $n=0,1,2, \ldots$.

From the estimate (4.13) we deduce that

$$
\left\|\left(\frac{d}{d \lambda}\right)^{n} \lambda\left(\lambda^{2}-A\right)^{-1} v\right\| \leq n !(\lambda-\omega)^{-n-1}\|v\| \text { for } v \in X \text { and } \lambda>\omega
$$

with arbitrary $\omega>0$. This estimate together with the equality

$$
\left(-\frac{d}{d \lambda}\right)^{n}\left(\lambda^{2}-A\right)^{-1} v=\sum_{k=0}^{n}\left(\begin{array}{l}
n \\
k
\end{array}\right) k ! \lambda^{-k-1}\left(-\frac{d}{d \lambda}\right)^{n-k} \lambda\left(\lambda^{2}-A\right)^{-1} v
$$

yields

$$
\left\|\left(\frac{d}{d \lambda}\right)^{n}\left(\lambda^{2}-A\right)^{-1} v\right\| \leq \omega^{-1} n !(\lambda-\omega)^{-n-1}\|v\|
$$

for $\lambda>\omega, v \in X$ and $n=0,1,2, \ldots$.

Differentiating (4.10) in $\lambda, n$-times and then in $x$ and noting that $w(0)=0$, we have

$$
\left|\left(\frac{d}{d x}\right)\left(-\frac{d}{d \lambda}\right)^{n}\left(\lambda^{2}-A\right)^{-1} v(x)\right| \leq \frac{n !}{\lambda^{n+1}}\|v\|
$$


for $\lambda>0, v \in X$ and $n=0,1,2, \ldots$. Moreover (4.11) implies

$$
\left|\left(-\frac{d}{d \lambda}\right)^{n} A\left(\lambda^{2}-A\right)^{-1} f(x)\right| \leq \frac{n !}{\lambda^{n+1}}\left\|f^{\prime}\right\|
$$

for $\lambda>0, f \in V$ and $n=0,1,2, \ldots$.

A simple computation shows that for $\lambda>\omega$, the operator $(\lambda-\mathcal{A})^{-1}$ exists and equals

$$
(\lambda-\mathcal{A})^{-1}=\left(\begin{array}{cc}
\lambda\left(\lambda^{2}-A\right)^{-1} & \left(\lambda^{2}-A\right)^{-1} \\
A\left(\lambda^{2}-A\right)^{-1} & \lambda\left(\lambda^{2}-A\right)^{-1}
\end{array}\right) .
$$

Thus by (4.13)-(4.17) we have

$$
\begin{aligned}
\left\|(\lambda-\mathcal{A})^{-n}\left(\begin{array}{l}
f \\
g
\end{array}\right)\right\|_{V \times X} & =\left\|\left(\frac{1}{(n-1) !}\right)\left(-\frac{d}{d \lambda}\right)^{n-1}(\lambda-\mathcal{A})^{-1}\left(\begin{array}{l}
f \\
g
\end{array}\right)\right\|_{V \times X} \\
& \leq\left(2+\omega^{-1}\right)(\lambda-\omega)^{-n}\left(\|f\|_{V}+\|g\|\right)
\end{aligned}
$$

for $\lambda>\omega,(f, g) \in V \times X$ and $n=0,1,2, \ldots$.

Acknowledgments. I would like to express my appreciation to Professor John A. Nohel for his useful comments. I also thank Professor Isao Miyadera for his constant encouragement and valuable advice.

\section{REFERENCES}

1. W. Arendt, Vector-valued Laplace transforms and Cauchy problems, Israel J. Math. 59 (1987), 327-352.

2. W. Arendt and H. Kellermann, Integrated solutions of Volterra integrodifferential equations and applications, in Volterra integrodifferential equations in Banach spaces and applications, Pitman Res. Notes in Math. Ser. 190 (1989), 21-51.

3. J. M. Ball, Strongly continuous semigroups, weak solutions and the variation of constants formula, Proc. Amer. Math. Soc. 63 (1977), 370-373.

4. G. Chen and R. Grimmer, Semigroups and integral equations, J. Integral Equations 2 (1980), 133-154.

5. G. Da Prato and E. Sinestrari, Differential operators with non dense domain, Ann. Scuola Norm, Sup. Pisa XIV (1987), 285-344.

6. W. Desch, R. Grimmer, and W. Schappacher, Some considerations for linear integrodifferential equations, J. Math. Anal. Appl. 104 (1984), 219-234.

7. N. Dunford and J. Schwartz, Linear operators, Part I, Interscience, Wiley, New York, 1958. 
8. R. Grimmer and H. Liu, Integrated semigroups and integrodifferential equations, Semigroup Forum 48 (1994), 79-95.

9. R. Grimmer and J. Prüss, On linear Volterra equations in Banach spaces, Comput. Math. Appl. 11 (1985), 189-205.

10. R. Grimmer and W. Schappacher, Weak solutions of integrodifferential equations and resolvent operators, J. Integral Equations 6 (1984), 205-229.

11. E. Hille and R. S. Phillips, Functional analysis and semigroups, Amer. Math. Soc. Colloq. Publ. 31 (1957).

12. H. Kellermann and M. Hieber, Integrated semigroups, J. Funct. Anal. 84 (1989), 160-180.

13. I. Miyadera, M. Okubo and N. Tanaka, $\alpha$-times integrated semigroups and abstract Cauchy problems, Memoirs of School of Science Engineering, Waseda University 57, Tokyo (1993), 267-289.

14. F. Neubrander, Well-posedness of higher order abstract Cauchy problems, Trans. Amer. Math. Soc. 295 (1986), 257-290.

15. - Integrated semigroups and their application to complete second order Cauchy problems, Semigroup Forum 38 (1989), 233-251.

16. A. Nogami, Integrated C-semigroups and the inhomogeneous Cauchy problems, Master's thesis, Waseda University, Tokyo (1991).

17. A. Pazy, Semigroups of linear operators and applications to partial differential equations, Springer-Verlag, New York, 1983.

18. H. Serizawa and M. Watanabe, Time-dependent perturbation for cosine families in Banach spaces, Houston J. Math. 12 (1986), 579-586.

19. H. R. Thieme, Semiflows generated by Lipschitz perturbations of non-densely defined operators, Differential Integral Equations 3 (1990), 1035-1066.

20. K. Tsuruta, On linear integro-differential equations in a Banach space, Tokyo J. Math. 3 (1980), 331-352.

Department of Mathematics, School of Education, Waseda University, 1-6-1 Nishi-Waseda, Shinjuku-Ku, Tokyo 169-50, Japan 